\title{
Contextual approach to quantum mechanics and the theory of the fundamental prespace
}

\author{
Andrei Khrennikov* \\ MSI, University of Växjö, S-35195, Sweden
}

November 1, 2018

\begin{abstract}
We constructed a Hilbert space representation of a contextual Kolmogorov model. This representation is based on two fundamental observables - in the standard quantum model these are the position and momentum observables. This representation has all distinguishing features of the quantum model. Thus in spite all "No-Go" theorems (e.g., von Neumann, Kochen and Specker,..., Bell) we found the realist basis of quantum mechanics. Our representation is not standard model with hidden variables. In particular, this is not a reduction of the quantum model to the classical one. Moreover, we see that such a reduction is even in principle impossible. This impossibility is not the consequence of a mathematical theorem but it follows from the physical structure of the model. By our model quantum states are very rough images of domains in the space of fundamental parameters - PRESPACE. Those domains represent complexes of physical conditions. By our model both classical and quantum physics describe REDUCTION of PRESPACE-INFORMATION. Quantum mechanics is not complete. In particular, there are prespace contexts which can be represented only by a so called hyperbolic quantum model. We predict violations of the Heisenberg's uncertainty principle and existence of dispersion free states.
\end{abstract}

*International Center for Mathematical Modeling in Physics and Cognitive Sciences, Andrei.Khrennikov@msi.vxu.se; supported by EU-Network "QP and Applications 


\section{Introduction}

Since the early days of quantum mechanics (QM) there have been permanent discussions on the problem:

\section{What is really QM about?}

In particular, the question of the greatest importance is

Can QM be interpreted in a realist way?

We recall that A. Einstein was sure that a realist interpretation of QM could be found: QM is not complete and there would be found finer, "prequantum", descriptions of physical reality. The most well known consideration of these problems was presented in the famous EPR paper [1]. This paper induced intensive discussions and, finally, various "no-go" theorems. The "final no-go theorem" of J. Bell [2] induced a rather common opinion that QM cannot be based on a local realist model. Personally I do not think that locality (in the physical space $\mathbf{R}^{3}$ ) really plays the fundamental role in the realist interpretation of $\mathrm{QM}^{1}$. Therefore I would like to come back to the original attempts of Einstein to find violations of Heisenberg's uncertainty relation. This was the crucial point of investigations of the possibility of a realist interpretation of QM. Unfortunately (as it was already mentioned) the main stream of those investigations was later directed to the EPR-Bohm-Bell framework.

We underline that the possibility to violate Heisenberg's uncertainty principle for two fundamental variables, e.g., position and momentum, need not imply the realization of the program of the hidden variables (HV) reconstruction of QM. The latter can be formulated (see J. Bell [4]):

"The question at issue is whether the quantum mechanical states can be regarded as ENSEMBLES of states further specified by additional variables, such that given values of these variables together with the state vector determine precisely the results of individual measurements."

Those hypothetical well-specified states are said to be 'dispersion free'. In this paper we present a model in which dispersion free states exist for two fundamental variables $b$ and $a$ (represented by noncommutative operators $\widehat{b}$ and $\widehat{a}$ ) but for an arbitrary quantum observable such states need not exist.

\footnotetext{
${ }^{1}$ Since this paper is not about EPR, I would no like to go deeply into detail. It should be remarked that it is impossible even to discuss locality in Bell's framework, because space variables do not present in Bell's model of "local reality" and Bell's inequality says nothing about those variables, see [3] for detail. Regarding the original EPR experiment I remark that a local realist model was recently constructed, see [3].
} 
Of course, an orthodox adherent of the HV-approach would not be so much interested in our model. But we could not do anything more. This is the reality.

Moreover, even the general ideology of HV reduction of QM has not so much to do with our approach. We are not looking for some HV-models for QM. In the HV-approach the main problem is not nonexistence of $\mathrm{HV}$ models, but existence of too many models. Many of them are totally meaningless from the physical viewpoint, see, e.g., Bell's example [1] for the two dimensional HV-system. The HV-reductionist approach has also induced numerous discussions which kinds of reductions are acceptable - von Neumann's "no-go" and Kochen and Specker's theorems (see [5], [6], and see [7]-[9] for reviews), De Broglie double solution model [10], Bohmian mechanics [11], [12], and Nelson-Guerra stochastic mechanics, see, e.g., [13].

I think that the starting point should be not QM. The QM-formalism by itself could not tell so much about features of a prequantum realist model which is (roughly) encoded by this formalism. Inversely we should start with a realist model and try to find a "natural representation" of such a model in a Hilbert space. Since QM is a statistical theory such a natural representation should be a probabilistic one. Roughly speaking the problem is to find a natural way to represent Kolmogorovian probabilities by complex amplitudes (or in the abstract framework by normalized vectors in a Hilbert space). In a series of papers [14] - [17] there was shown that such a representation can be constructed on the basis of a so called contextual formula of total probability for observables $b$ and $a$ :

$$
\begin{gathered}
p_{C}^{b}(x)=\sum_{y} p_{C}^{a}(y) p^{b / a}(x / y)+ \\
2 \sum_{y_{1}<y_{2}} \sqrt{p_{C}^{a}\left(y_{1}\right) p_{C}^{a}\left(y_{2}\right) p^{b / a}\left(x / y_{1}\right) p^{b / a}\left(x / y_{2}\right)} \cos \theta_{C}^{\left(y_{1} y_{2}\right)}(x),
\end{gathered}
$$

where $p_{C}^{a}(y)=\mathbf{P}(a=y / C), p_{C}^{b}(x)=\mathbf{P}(b=x / C)$ are probabilities to observe values $a=y$ and $b=x$ under the complex of physical conditions context $-C$ and $p^{b / a}(x / y)=\mathbf{P}(b=x / a=y)$ are transition probabilities. A complex amplitude $\varphi_{C}^{b / a}(x)$ corresponding to the representation (11) gives the QM-representation of context $C$. In [17] it was shown that for a Kolmogorov probability space $\mathcal{K}=(\Omega, \mathcal{F}, \mathbf{P})$ and a pair of incompatible Kolmogorovian random variables $b$ and $a$ we can construct a natural quantum representation. This representation is rigidly based on a pair of variables $b$ and $a$ 
- fundamental (for that concrete representation of physical reality) observables. In particular, the standard quantum representation is based on the position and momentum observables. There exists a map $J^{b / a}$ which maps contexts (represented by subsets of $\Omega$ ) into quantum states $\equiv$ complex $b / a$-transition amplitudes of probability ${ }^{2}$.

Points of $\Omega$ are interpreted as fundamental physical parameters ${ }^{3}$. We call $\Omega$ prespace and fundamental parameters - prepoints.

The main distinguishing feature of the representation map $J^{b / a}$ is the huge compression of information. In particular, every point represented in the conventional mathematical model of physical space by a vector $x \in \mathbf{R}^{3}$ is the image of a subset

$$
B_{x}=\{\omega \in \Omega: b(\omega)=x\}
$$

of $\Omega$ which can contain millions of prepoints. In the conventional quantum representation of the prespace the fundamental variable $b=q$ is the position observable. We have a similar picture for the momentum observable. In the quantum model we consider "classical physical points" $x \in \mathbf{R}^{3}$ as represented by eigenvectors of the position operator. Thus by going from the "classical physical space" $\mathbf{R}^{3}$ to the quantum physical (Hilbert) space $H$ and then to the prespace $\Omega$ we obtain finer and finer descriptions of reality.

Another distinguishing feature of the $J^{b / a}$-representation of the prespace $\Omega$ in the Hilbert space $H$ is the creation of superpositions of "classical states". The origin of the quantum superposition can be very easily explained by our prespace model. For example, let us consider a context $C \subset \Omega$ such that $C \subset B_{x_{1}} \cup B_{x_{2}}, x_{1}, x_{2} \in \mathbf{R}^{3}, x_{1} \neq x_{2}$, but neither $C \subset B_{x_{1}}$ nor $C \subset B_{x_{2}}$. The image $\varphi_{C}=J^{b / a}(C) \in H$ is a quantum state describing a quantum system which is "in a superposition of the positions" $x_{1}$ and $x_{2}$.

Thus inspite of all "no-go" theorems (e.g., von Neumann, Kochen and Specker, Bell, ...), we have constructed a realist model of QM. In this model (as it was wanted by A. Einstein) the Heisenberg uncertainty relation can be violated for fundamental observables (e.g., the position and momen-

\footnotetext{
${ }^{2}$ In some sense we came back to the original Hilbert's viewpoint to a wave function as a transition amplitude, see [18], see also Lande [19], cf. Gudder [20], Accardi [21], Ballentine [22], Khrennikov [14]-[17].

${ }^{3}$ If you like HV... But the general HV-approach was so discredited by former investigations (since people wanted too much for such a HV-description) that we would not like even to refer to HV.
} 
tum) which are used for our classical and quantum representations of reality. Points $(\omega \in \Omega)$ of the prespace are dispersion free states.

In our model only the fundamental observables correspond to random variables on the prespace. Other quantum and classical observables have only some indirect relation to random variables on the prespace. So we could not consider such, e.g., quantum observables as real observables - functions of fundamental parameters. Nevertheless, for a wide class of quantum observables (including QM-Hamiltonians) we have the coincidence of averages with averages of corresponding random variables on $\Omega$. Here we speak about averages with respect to the state $\varphi_{C}=J^{b / a}(C)$ and context $C$, respectively. In our model only quantum observables belonging to a special class (class $\left.\mathcal{O}_{+}(a, b)\right)$ have realist interpretation.

We underline that our investigations has nothing to do with attempts to find some general probabilistic model which would contain Kolmogorov as well as quantum probabilities as particular cases, cf., e.g., Mackey [24], Gudder [20], Ludwig [25], Devies and Lewis [26], Accardi [21], Ballentine [22] ..., Khrennikov [27], Hardy [28]. For us the main distinguishing feature of quantum theory is not a new ("quantum") behaviour of probabilities, but a special way of representation of (ordinary) probability.

By our model dispersion free states (for, e.g., position and momentum observables) can exist and the Heisenberg's uncertainty principle can be violated.

\section{Contextual formula of total probability}

Let $(\Omega, \mathcal{F}, \mathbf{P})$ be a Kolmogorov probability space, [29].

By the standard Kolmogorov axiomatics sets $A \in \mathcal{F}$ represent events. In our simplest model of contextual probability (Kolmogorovian contextual model) the same system of sets, $\mathcal{F}$, is used to represent complexes of experimental physical conditions - contexts. We can consider a set $C \in \mathcal{F}$ as a collection of physical parameters $\omega$ describing a complex of physical conditions. This is a context-interpretation of sets $C \in \mathcal{F}$.

By the event-interpretation of sets $A \in \mathcal{F}$ such a set $A$ is a collection of physical parameters inducing the corresponding event (denoted by the same symbol $A$ ).

In principle, in a mathematical model events and contexts can be represented by different families of sets, see, e.g., Renye's model. We will not do 
this from the beginning. But later we will fix a proper subfamily of contexts $\mathcal{C} \subset \mathcal{F}$.

The conditional probability is mathematically defined by the Bayes' formula:

$$
\mathbf{P}(A / C)=\frac{\mathbf{P}(A C)}{\mathbf{P}(C)}, \mathbf{P}(C) \neq 0 .
$$

In our contextual model this probability has the meaning of the probability of occurrence of the event $A$ under the complex of physical conditions $C$. Thus it is not the probability of occurrence of the event $A$ under the condition that the event $C$ has occurred (as it is assumed in the Kolmogorov theory). ${ }^{4}$

Thus it would be more natural to call $\mathbf{P}(A / C)$ a contextual probability and not conditional probability. Roughly speaking to find $\mathbf{P}(A / C)$ we should find parameters $\omega^{A}$ favouring to the occurrence of the event $A$ among parameters $\omega^{C}$ describing the complex of physical conditions $C$.

Let $\mathcal{A}=\left\{A_{n}\right\}$ be finite or countable complete group of inconsistent contexts:

$$
A_{i} A_{j}=\emptyset, i \neq j, \quad \cup_{i} A_{i}=\Omega .
$$

Let $B \in \mathcal{F}$ be an event and $C \in \mathcal{F}$ be a context and let $\mathbf{P}(C)>0$. We have the standard formula of total probability, see, e.g., [17]:

$$
\mathbf{P}(B / C)=\frac{\mathbf{P}(B C)}{\mathbf{P}(C)}=\sum_{n} \frac{\mathbf{P}\left(B A_{n} C\right) \mathbf{P}\left(A_{n} C\right)}{\mathbf{P}(C) \mathbf{P}\left(A_{n} C\right)}
$$

(if $\mathbf{P}\left(A_{n} C\right)>0$ for all $n$ ) and hence

$$
\mathbf{P}(B / C)=\sum_{n} \mathbf{P}\left(A_{n} / C\right) \mathbf{P}\left(B / A_{n} C\right)
$$

Of course, in the conventional Kolmogorov model we operate only with events. Thus inspite of using the standard Kolmogorov measure-theoretical probabilistic formalism, from the very beginning we use a new interpretation of conditioning in this formalism. Instead of the conventional evenconditioning, we use context-conditioning. Thus there is nothing new from the mathematical viewpoint and the reader may be curious: Is it possible to find something new by using the same mathematical apparatus and by changing only the interpretation? Yes, we shall construct totally new

\footnotetext{
${ }^{4}$ The reader might think that the difference in interpretations is negligible. But I would like to underline that this is the crucial point of all our considerations.
} 
representation of the Kolmogorov model in a Hilbert space. This representation is nontrivial - Kolmogorovian (but contextual) random variables are represented by in general noncommutative operators.

In particular, let $a$ and $b$ be discrete random variables taking values $a_{i}, i=$ $1, \ldots, k_{a}$ and $b_{j}, j=1, \ldots, k_{b}$, where $k_{a}, k_{b}<\infty$. We have

$$
\mathbf{P}\left(b=b_{i} / C\right)=\sum_{n} \mathbf{P}\left(a=a_{n} / C\right) \mathbf{P}\left(b=b_{i} / a=a_{n}, C\right) .
$$

Let a measurement of the variable $a$ disturb essentially physical systems $\omega \in \Omega$. Let us fix some complex of conditions (context) $C$, see [14]-[17] for detail. One cannot measure $b$ and $a$ simultaneously in the context $C$. Thus the probabilities $\mathbf{P}\left(b=b_{i} / a=a_{n}, C\right)$ are "hidden" (or ontic) probabilities. ${ }^{5}$ However, we can measure the variable $b$ in the context $A_{n}=\left\{\omega: a(\omega)=a_{n}\right\}$. Thus we can not prepare for the context $C$ systems $\omega$ such that we know that simultaneously $b(\omega)=b_{i}, a(\omega)=a_{n}$, but we can prepare systems $\omega$ such that $a(\omega)=a_{n}$ and in this context we can perform the $b$-measurement. Hence the probabilities $\mathbf{P}\left(b=b_{i} / a=a_{n}\right)=\mathbf{P}\left(B_{i} / A_{n}\right)$ are well defined. Here

$$
B_{i}=\left\{\omega \in \Omega: b(\omega)=b_{i}\right\} \text { and } A_{n}=\left\{\omega \in \Omega: a(\omega)=a_{n}\right\} .
$$

I would like to modify the formula of total probability (2) by eliminating hidden probabilities $\mathbf{P}\left(b=b_{i} / a=a_{n}, C\right)$ and using only observable probabilities $\mathbf{P}\left(b=b_{i} / a=a_{n}\right)$.

Definition 1. (Context) $A$ set $C$ belonging to $\mathcal{F}$ is said to be a context with respect to a complete group of inconsistent contexts $\mathcal{A}=\left\{A_{n}\right\}$ if $\mathbf{P}\left(A_{n} C\right) \neq 0$ for all $n$.

We denote the set of all $\mathcal{A}$-contexts by the symbol $\mathcal{C}_{\mathcal{A}}$.

Definition 2. Let $\mathcal{A}=\left\{A_{n}\right\}$ and $\mathcal{B}=\left\{B_{n}\right\}$ be two complete groups of inconsistent contexts. They are said to be incompatible if $\mathbf{P}\left(B_{n} A_{k}\right) \neq 0$ for all $n$ and $k$.

Thus $\mathcal{B}$ and $\mathcal{A}$ are incompatible iff every $B_{n}$ is a context with respect to $\mathcal{A}$ and vice versa, see Appendix 1 for detail.

Random variables $a$ and $b$ inducing incompatible complete groups $\mathcal{A}=$ $\left\{A_{n}\right\}$ and $\mathcal{B}=\left\{B_{k}\right\}$ of inconsistent contexts are said to be incompatible random variables.

\footnotetext{
${ }^{5}$ We are not able to select parameters $\omega^{A_{n}}$ favouring to the realization of $a=a_{n}$ without to disturb context $C$.
} 
Theorem 1. (Interference formula of total probability) Let $\mathcal{A}$ and $\mathcal{B}$ be incompatible and let $C$ be a context with respect to $\mathcal{A}$. Then the following "interference formula of total probability" holds true for any $B \in \mathcal{B}$ :

$$
\begin{gathered}
\mathbf{P}(B / C)=\sum \mathbf{P}\left(A_{n} / C\right) \mathbf{P}\left(B / A_{n}\right)+ \\
2 \sum_{n<m} \lambda_{n m}(B / \mathcal{A}, C) \sqrt{\mathbf{P}\left(A_{n} / C\right) \mathbf{P}\left(A_{m} / C\right) \mathbf{P}\left(B / A_{n}\right) \mathbf{P}\left(B / A_{m}\right)}
\end{gathered}
$$

where

$$
\lambda_{n m}(B / \mathcal{A} ; C)=\frac{\delta_{n m}(B / \mathcal{A} ; C)}{2 \sqrt{\mathbf{P}\left(A_{n} / C\right) \mathbf{P}\left(B / A_{n}\right) \mathbf{P}\left(A_{m} / C\right) \mathbf{P}\left(B / A_{m}\right)}}
$$

and

$$
\begin{aligned}
& \delta_{n m}(B / \mathcal{A} ; C) \\
= & \frac{\left[\mathbf{P}\left(A_{n} / C\right)\left(\mathbf{P}\left(B / A_{n} C\right)-\mathbf{P}\left(B / A_{n}\right)\right)+\mathbf{P}\left(A_{m} / C\right)\left(\mathbf{P}\left(B / A_{m} C\right)-\mathbf{P}\left(B / A_{m}\right)\right]\right.}{k_{a}-1}
\end{aligned}
$$

Proof. We have:

$$
\begin{aligned}
\mathbf{P}(B / C)= & \sum_{n} \mathbf{P}\left(A_{n} / C\right)\left(\mathbf{P}\left(B / A_{n} C\right)+\mathbf{P}\left(B / A_{n}\right)-\mathbf{P}\left(B / A_{n}\right)\right) \\
& =\sum_{n} \mathbf{P}\left(A_{n} / C\right) \mathbf{P}\left(B / A_{n}\right)+\delta(B / \mathcal{A}, C),
\end{aligned}
$$

where

$$
\delta(B / \mathcal{A}, C)=\sum_{n} \mathbf{P}\left(A_{n} / C\right)\left(\mathbf{P}\left(B / A_{n} C\right)-\mathbf{P}\left(B / A_{n}\right)\right) .
$$

Finally, we remark that we can represent the perturbation term as the sum of perturbation terms corresponding to pairs of $\left(A_{n}, A_{m}\right)$ :

$$
\delta(B / \mathcal{A}, C)=\sum_{n<m} \delta_{n m}(B / \mathcal{A} ; C),
$$

where $\delta_{n m}(B / \mathcal{A} ; C)$ is given by (4).

The $\lambda_{n m}(B / \mathcal{A}, C)$ are called coefficients of statistical disturbance. Coefficients $\lambda_{n m}(B / \mathcal{A}, C)$ describe disturbances of probabilities induced by filtrations with respect to values $a=a_{n}$ in the context $C$. Depending on magnitudes of these coefficients we can rewrite the nonconventional formula of 
total probability in various forms that are useful for representing (3) as a transformation in a complex linear space or a Clifford modular, see [14]-[17] for the details.

In our further investigations we will use the following result:

Lemma 1. Let conditions of Corollary 1. hold true. Then

$$
\sum_{k} \delta\left(B_{k} / \mathcal{A}, C\right)=0
$$

Proof. We have $1=\sum_{k} \mathbf{P}\left(B_{k} / C\right)=\sum_{k} \sum_{n} \mathbf{P}\left(A_{n} / C\right) \mathbf{P}\left(B_{k} / A_{n}\right)+$ $\sum_{k} \delta\left(B_{k} / \mathcal{A}, C\right)$. But $\sum_{n}\left(\sum_{k} \mathbf{P}\left(B_{k} / A_{n}\right)\right) \mathbf{P}\left(A_{n} / C\right)=1$.

As a consequence of this lemma we have:

$$
\sum_{k} \sum_{l<m} \lambda_{l m}\left(B_{k} / \mathcal{A}, C\right) \sqrt{\mathbf{P}\left(A_{l} / C\right) \mathbf{P}\left(A_{m} / C\right) \mathbf{P}\left(B_{k} / A_{l}\right) \mathbf{P}\left(B_{k} / A_{m}\right)}=0
$$

1). Suppose that $a=a_{n}$ filtrations (in the context $\left.C\right)^{6}$ induce statistical disturbances having relatively small coefficients $\lambda_{n m}(B / \mathcal{A}, C)$, namely, for every $B \in \mathcal{B}$

$$
\left|\lambda_{n m}(B / \mathcal{A}, C)\right| \leq 1
$$

In this case we can introduce new statistical parameters $\theta_{n m}(B / \mathcal{A}, C) \in$ $[0, \pi]$ and represent the coefficients of statistical disturbance in the trigonometric form:

$$
\lambda_{n m}(B / \mathcal{A}, C)=\cos \theta_{n m}(B / \mathcal{A}, C) .
$$

Parameters $\theta_{n m}(B / \mathcal{A}, C)$ are said to be relative phases of an event $B$ with respect to a complete group of inconsistent events $\mathcal{A}$ (in the context $C$ ).

In this case we obtain the following interference formula of total probability:

$$
\begin{gathered}
\mathbf{P}(B / C)=\sum_{n} \mathbf{P}\left(A_{n} / C\right) \mathbf{P}\left(B / A_{n}\right) \\
+2 \sum_{n<m} \cos \theta_{n m}(B / \mathcal{A}, C)(B / \mathcal{A}, C) \sqrt{\mathbf{P}\left(A_{n} / C\right) \mathbf{P}\left(A_{m} / C\right) \mathbf{P}\left(B / A_{n}\right) \mathbf{P}\left(B / A_{m}\right)} .
\end{gathered}
$$

\footnotetext{
${ }^{6}$ First we prepare a statistical ensemble $O_{C}$ of physical systems $\omega$ under the complex of (e.g., physical) conditions $C$. Then we perform a measurement of the random variable $a$ for elements of the ensemble $O_{C}$. Finally, we select all systems for which we obtained the value $a=a_{n}$.
} 
This is nothing other than the famous formula of interference of probabilities. $^{7}$ We demonstrated that in the opposite of the common (especially in quantum physics) opinion nontrivial interference of probabilities need not be related to some non-Kolmogorovian features of a probabilistic model. In our considerations everything is Kolmogorovian. Interference of probabilities is a consequence of the impossibility of using conditioning with respect to $\left\{a=a_{n}, C\right\}$ (to combine two contexts $-C$ and $a$ ) for random variables $a$ which measurement disturbs essentially physical systems $\omega \in \Omega$.

Starting from (8) we shall derive (for dichotomous random variables) Born's rule, construct for any context $C$ a complex probability amplitude, introduce a Hilbert space structure on the space of complex amplitudes and represent random variables on the Kolmogorov probability space by (in general noncommutative) operators in the Hilbert space.

$2)$. Suppose that $a=a_{n}$ filtrations induce statistical disturbances having relatively large coefficients $\lambda_{n m}(B / \mathcal{A}, C)$, namely, for every $B \in \mathcal{B}$

$$
\left|\lambda_{n m}(B / \mathcal{A}, C)\right| \geq 1 \text {. }
$$

In this case we can introduce new statistical parameters $\theta_{n m}(B / \mathcal{A}, C) \in$ $[0,+\infty]$ and represent the coefficients of statistical disturbance in the trigonometric form:

$$
\lambda_{n m}(B / \mathcal{A}, C)= \pm \cosh \theta_{n m}(B / \mathcal{A}, C) .
$$

Parameters $\theta_{n m}(B / \mathcal{A}, C)$ are said to be hyperbolic relative phases of an event $B$ with respect to a complete group of inconsistent events $\mathcal{A}$ (in the context $C)$.

In this case we obtain the following interference formula of total probability:

$$
\begin{gathered}
\mathbf{P}(B / C)=\sum_{n} \mathbf{P}\left(A_{n} / C\right) \mathbf{P}\left(B / A_{n}\right) \\
\pm 2 \sum_{n<m} \cosh \theta_{n m}(B / \mathcal{A}, C)(B / \mathcal{A}, C) \sqrt{\mathbf{P}\left(A_{n} / C\right) \mathbf{P}\left(A_{m} / C\right) \mathbf{P}\left(B / A_{n}\right) \mathbf{P}\left(B / A_{m}\right)} .
\end{gathered}
$$

\footnotetext{
${ }^{7}$ Typically this formula is derived by using the Hilbert space (unitary) transformation corresponding to the transition from one orthonormal basis to another and Born's probability postulate. The orthonormal basis under quantum consideration consist of eigenvectors of operators (noncommutative) corresponding to quantum physical observables $a$ and $b$.
} 
3). Suppose that $a=a_{n}$ filtrations induce for some $n$ statistical disturbances having relatively small coefficients $\lambda_{n m}(B / \mathcal{A}, C)$ and for other $n$ statistical disturbances having relatively large coefficients $\lambda_{n m}(B / \mathcal{A}, C)$. Here we have the interference formula of total probability containing trigonometric as well as hyperbolic interference terms.

\section{Dichotomous random variables.}

We study only models with trigonometric interference. We set

$$
\mathcal{C}=\left\{C \in \mathcal{C}_{\mathcal{A}}:\left|\lambda\left(B_{j} / \mathcal{A}, C\right)\right| \leq 1\right\}
$$

We call elements of $\mathcal{C}$ trigonometric contexts. We shall see that QM can be interpreted as a representation of trigonometric contexts. We can also introduce hyperbolic contexts which can be represented in a hyperbolic Hilbert space, see [30].

3.1. Interference and complex probability amplitude, Born's rule. Let us study in more detail the case of incompatible dichotomous random variables $a=a_{1}, a_{2}, b=b_{1}, b_{2}$. We set $Y=\left\{a_{1}, a_{2}\right\}, X=\left\{b_{1}, b_{2}\right\}$ ("spectra" of random variables $a$ and $b$ ). Let $C \in \mathcal{C}$ be a context for both random variables $a$ and $b$. We set

$$
p_{C}^{a}(y)=\mathbf{P}(a=y / C), p_{C}^{b}(x)=\mathbf{P}(b=x / C), p(x / y)=\mathbf{P}(b=x / a=y),
$$

$x \in X, y \in Y$. The interference formula of total probability (8) can be written in the following form

$$
p_{c}^{b}(x)=\sum_{y \in Y} p_{C}^{a}(y) p(x / y)+2 \cos \theta_{C}(x) \sqrt{\Pi_{y \in Y} p_{C}^{a}(y) p(x / y)},
$$

where $\theta_{C}(x)=\theta(b=x / \mathcal{A}, C)=\arccos \lambda(b=x / \mathcal{A}, C), x \in X, C \in \mathcal{C}$. We remark that in the case of dichotomous random variables:

$$
\delta(b=x / \mathcal{A}, C)=p_{c}^{b}(x)-\sum_{y \in Y} p_{C}^{a}(y) p(x / y)
$$

and

$$
\lambda(b=x / \mathcal{A}, C)=\frac{\delta(b=x / \mathcal{A}, C)}{2 \sqrt{\Pi_{y \in Y} p_{C}^{a}(y) p(x / y)}} .
$$


By using the elementary formula:

$$
D=A+B+2 \sqrt{A B} \cos \theta=\left|\sqrt{A}+e^{i \epsilon \theta} \sqrt{B}\right|^{2}
$$

for $A, B>0, \epsilon= \pm 1, \theta \in[0, \pi]$. we can represent the probability $p_{C}^{b}(x)$ as the square of the complex amplitude:

$$
p_{C}^{b}(x)=\left|\varphi_{C}(x)\right|^{2} .
$$

We fix some pair of signs $\epsilon(x), x \in X$ (e.g., $\epsilon\left(b_{1}\right)=-1$ and $\epsilon\left(b_{2}\right)=+1$ ). We set

$$
\varphi(x) \equiv \varphi_{C}(x)=\sqrt{p_{C}^{a}\left(a_{1}\right) p\left(x / a_{1}\right)}+e^{\epsilon(x) \theta_{C}(x)} \sqrt{p_{C}^{a}\left(a_{2}\right) p\left(x / a_{2}\right)} .
$$

We denote the space of functions: $\varphi: X \rightarrow \mathbf{C}$ by the symbol $E=\Phi(X, \mathbf{C})$. Since $X=\left\{b_{1}, b_{2}\right\}$, the $E$ is the two dimensional complex linear space. Dirac's $\delta$-functions $\left\{\delta\left(b_{1}-x\right), \delta\left(b_{2}-x\right)\right\}$ form the canonical basis in this space. For each $\varphi \in E$ we have

$$
\varphi(x)=\varphi\left(b_{1}\right) \delta\left(b_{1}-x\right)+\varphi\left(b_{2}\right) \delta\left(b_{2}-x\right) .
$$

By using the representation (12) we construct the map

$$
J^{b / a}: \mathcal{C} \rightarrow \Phi(X, \mathbf{C})
$$

The $J^{b / a}$ maps contexts (complexes of, e.g., physical conditions) into complex amplitudes. The representation (11) of probability as the square of the absolute value of the complex $(b / a)$-amplitude is nothing other than the famous Born rule.

Remark 1. We underline that the complex linear space representation (12) of the set of contexts $\mathcal{C}$ is based on a pair $(a, b)$ of incompatible (Kolmogorovian) random variables. Here $\varphi_{C}=\varphi_{C}^{b / a}$.

The complex amplitude $\varphi_{C}(x)$ can be called a wave function of the complex of physical conditions, context $C$, cf [14]- [16], of a pure state.

We recall that we obtained complex probability amplitudes in the conventional Kolmogorov framework without appealing to the standard wave or Hilbert space arguments. As we shall see, the map $J^{b / a}$ gives a quantumlike representation of conventional Kolmogorov probability model. 
In principle, we can represent each context $C \in \mathcal{C}$ by a family of complex amplitudes:

$$
\varphi(x) \equiv \varphi_{C}(x)=\sum_{y \in Y} \sqrt{p_{C}^{a}(y) p(x / y)} e^{i \xi_{C}(x / y)}
$$

such that

$$
\xi_{C}\left(x / a_{1}\right)-\xi_{C}\left(x / a_{2}\right)=\theta_{C}(x) .
$$

For such complex amplitudes we also have Born's rule (11). However, to simplify considerations we shall consider only the representation (12) and the map (13) induced by this representation.

3.2. Hilbert space representation of Born's rule. We set

$$
e_{x}^{b}(\cdot)=\delta(x-\cdot)
$$

The representation (11) can be rewritten in the following form:

$$
p_{C}^{b}(x)=\left|\left(\varphi_{C}, e_{x}^{b}\right)\right|^{2},
$$

where the scalar product in the space $E=\Phi(X, C)$ is defined by the standard formula:

$$
(\varphi, \psi)=\sum_{x \in X} \varphi(x) \bar{\psi}(x)
$$

The system of functions $\left\{e_{x}^{b}\right\}_{x \in X}$ is an orthonormal basis in the Hilbert space $H=(E,(\cdot, \cdot))$

Let $X \subset R$. By using the Hilbert space representation of Born's rule (15) we obtain the Hilbert space representation of the expectation of the (Kolmogorovian) random variable $b$ :

$$
E(b / C)=\sum_{x \in X} x p_{C}^{b}(x)=\sum_{x \in X} x\left|\varphi_{C}(x)\right|^{2}=\left(\hat{b} \varphi_{C}, \varphi_{C}\right)
$$

where $\hat{b}: \Phi(X, \mathbf{C}) \rightarrow \Phi(X, \mathbf{C})$ is the multiplication operator. This operator can also be determined by its eigenvectors: $\hat{b} e_{x}^{b}=x e_{x}^{b}, x \in X$.

We set

$u_{j}^{a}=\sqrt{p_{C}^{a}\left(a_{j}\right)}, u_{j}^{b}=\sqrt{p_{C}^{b}\left(b_{j}\right)}, p_{i j}=p\left(b_{j} / a_{i}\right), u_{i j}=\sqrt{p_{i j}}, \theta_{j}=\theta_{C}\left(b_{j}\right), \epsilon_{j}=\epsilon\left(b_{j}\right)$. 
We remark that the coefficients $u_{j}^{a}, u_{j}^{b}$ depend on a context $C$; so $u_{j}^{a}=$ $u_{j}^{a}(C), u_{j}^{b}=u_{j}^{b}(C)$. We also consider the matrix of transition probabilities $\mathbf{P}^{b / a}=\left(p_{i j}\right)$. It is always a stochastic matrix. ${ }^{8}$ We have, see (14), that

$$
\varphi_{C}=v_{1}^{b} e_{1}^{b}+v_{2}^{b} e_{2}^{b} \text {, where } v_{j}^{b}=u_{1}^{a} u_{1 j}+u_{2}^{a} u_{2 j} e^{i \epsilon_{j} \theta_{j}} .
$$

So

$$
p_{C}^{b}\left(b_{j}\right)=\left|v_{j}^{b}\right|^{2}=\left|u_{1}^{a} u_{1 j}+u_{2}^{a} u_{2 j} e^{i \epsilon_{j} \theta_{j}}\right|^{2} .
$$

This is the interference representation of probabilities that is used, e.g., in quantum formalism. ${ }^{9}$ We recall that we obtained (17) starting with the interference formula of total probability, (10).

3.3. Born's rule and Hilbert space representations. We would like to obtain (17) by using the standard quantum procedure, namely, transition from the orthonormal basis $\left\{e_{j}^{b}\right\}$ corresponding the $b$-variable to a new basis $\left\{e_{j}^{a}\right\}$ which corresponds to the $a$-variable. Thus we would like to have Born's rule not only in the $b$-representation, but also in the $a$-representation. As we shall see, we cannot be lucky in the general case. Starting from two arbitrary incompatible (Kolmogorovian) random variables $a$ and $b$ we obtained a complex linear space representation of the probabilistic model which is essentially more general than the standard quantum representation. In our (more general) linear representation the "dual variable" a need not be represented by a symmetric operator (matrix) in the Hilbert space $H$ generated by the $b$.

For any context $C_{0}$, we can represent the $\varphi=\varphi_{C_{0}}$ in the form:

$$
\varphi=u_{1}^{a} e_{1}^{a}+u_{2}^{a} e_{2}^{a}
$$

where

$$
e_{1}^{a}=\left(u_{11}, u_{12}\right) \quad e_{2}^{a}=\left(e^{i \epsilon_{1} \theta_{1}} u_{21}, \quad e^{i \epsilon_{2} \theta_{2}} u_{22}\right)
$$

Here $\left\{e_{i}^{a}\right\}$ is a system of vectors in $E$ corresponding to the $a$-observable. We suppose that vectors $\left\{e_{i}^{a}\right\}$ are lineary independent, so $\left\{e_{i}^{a}\right\}$ is a basis in $E$. We have:

$$
e_{1}^{a}=v_{11} e_{1}^{b}+v_{12} e_{2}^{b}, \quad e_{2}^{a}=v_{21} e_{1}^{b}+v_{22} e_{2}^{b}
$$

Here $V=\left(v_{i j}\right)$ is the matrix corresponding to the transformation of complex amplitudes: $v_{11}=u_{11}, v_{21}=u_{21}$ and $v_{12}=e^{i \epsilon_{1} \theta_{1}} u_{21}, v_{22}=e^{i i \epsilon_{2} \theta_{2}} u_{22}$.

\footnotetext{
${ }^{8}$ So $p_{i 1}+p_{i 2}=1, i=1,2$.

${ }^{9}$ By starting with the general representation (14) we obtain $v_{j}^{b}=u_{1}^{a} u_{1 j} e^{i \xi_{1 j}}+u_{2}^{a} u_{2 j} e^{i \xi_{2 j}}$ and the interference representation $p_{C}^{b}\left(b_{j}\right)=\left|v_{j}^{b}\right|^{2}=\left|u_{1}^{a} u_{1 j} e^{i \xi_{1 j}}+u_{2}^{a} u_{2 j} e^{i \xi_{2 j}}\right|^{2}$.
} 
We would like to find a class of matrixes $V$ such that Born's rule (in the Hilbert space form), see (15), holds true also in the $a$-basis:

$$
p_{C}^{a}\left(a_{j}\right)=\left|\left(\varphi, e_{j}^{a}\right)\right|^{2} .
$$

By (18) we have Born's rule iff $\left\{e_{i}^{a}\right\}$ was an orthonormal basis, i.e., the $V$ is a unitary matrix. Since we study the two-dimensional case (i.e., dichotomous random variables), $V \equiv V^{b / a}$ is unitary iff the matrix of transition probabilities $\mathbf{P}^{b / a}$ is double stochastic. ${ }^{10}$

However, there is some difficulty. In fact, we constructed the $a$-basis starting with one fixed context $C_{0}$. The basis $e_{j}^{a}$ depends on $C_{0}$ (via the phases $\left.\theta_{C_{0}}(x)\right): e_{j}^{a}=e_{j}^{a}\left(C_{0}\right)$. In principle, the validity of Born's rule for the context $C_{0}$ in the basis $e_{j}^{a}\left(C_{0}\right)$ need not imply this rule for any context $C$ in the same basis $e_{j}^{a}\left(C_{0}\right)$. We shall see that for double stochastic matrices of transition probabilities (and only such matrices) we can really construct the $a$-representation starting with some fixed $C_{0}$. However, we should choose signs $\epsilon(x)$ in the representation (12) in a special way. We recall that the map $J^{b / a}$ was constructed for fixed signs $\epsilon_{1}$ and $\epsilon_{2}$; so $J^{b / a}=J^{b / a}\left(\epsilon_{1}, \epsilon_{2}\right)$.

We now investigate this problem. We remind that we constructed the matrix $V$ by using the fixed context $C_{0}$, so $V=V\left(C_{0}\right)$. For any $C \in \mathcal{C}$, we would like to represent the wave function as

$$
\phi_{C}=v_{1}^{a}(C) e_{1}^{a}\left(C_{0}\right)+v_{2}^{a}(C) e_{2}^{a}\left(C_{0}\right) \text {, where }\left|v_{j}^{a}(C)\right|^{2}=p_{C}^{a}\left(a_{j}\right) .
$$

It is clear that, for any $C \in \mathcal{C}$, we can represent the wave function as

$$
\begin{aligned}
& \phi_{C}\left(b_{1}\right)=u_{1}^{a}(C) v_{11}\left(C_{0}\right)+e^{i \epsilon_{1}\left[\theta_{C}\left(b_{1}\right)-\theta_{C_{0}}\left(b_{1}\right)\right]} u_{2}^{a}(C) v_{12}\left(C_{0}\right) \\
& \phi_{C}\left(b_{2}\right)=u_{1}^{a}(C) v_{21}\left(C_{0}\right)+e^{i \epsilon_{1}\left[\theta_{C}\left(b_{2}\right)-\theta_{C_{0}}\left(b_{2}\right)\right]} u_{2}^{a}(C) v_{22}\left(C_{0}\right)
\end{aligned}
$$

Thus to obtain (20) we should have:

$$
\epsilon_{1}\left[\theta_{C}\left(b_{1}\right)-\theta_{C_{0}}\left(b_{1}\right)\right]=\epsilon_{2}\left[\theta_{C}\left(b_{2}\right)-\theta_{C_{0}}\left(b_{2}\right](\bmod 2 \pi)\right.
$$

for any pair of contexts $C_{0}$ and $C_{1}$. Thus

$$
\Delta(C)=\epsilon_{1} \theta_{C}\left(b_{1}\right)-\epsilon_{2} \theta_{C}\left(b_{2}\right)=\Delta
$$

should be a constant $(\bmod 2 \pi)$ on $\mathcal{C}$.

\footnotetext{
${ }^{10}$ So it is stochastic and, moreover, $p_{1 j}+p_{2 j}=1, j=1,2$.
} 


\subsection{The role of the condition of double stochasticity.}

Lemma 2. Let $a$ and $b$ be incompatible random variables and let the matrix of transition probabilities $\mathbf{P}^{b / a}$ be double stochastic. Then:

$$
\cos \theta_{C}\left(b_{1}\right)=-\cos \theta_{C}\left(b_{2}\right)
$$

for any context $C \in \mathcal{C}$.

Proof. By Lemma 1 we have:

$$
\sum_{x \in X} \cos \theta_{C}(x) \sqrt{\Pi_{y \in Y} p_{c}^{a}(y) p(x / y)}=0
$$

But for a double stochastic matrix $(p(x / y))$ we have:

$$
\Pi_{y \in Y} p_{c}^{a}\left(a_{1}\right) p\left(b_{1} / y\right)=\Pi_{y \in Y} p_{c}^{a}\left(a_{2}\right) p\left(b_{2} / y\right) .
$$

Since random variables $a$ and $b$ are incompatible, we have $p(x / y) \neq 0, x \in$ $X, y \in Y$. Since $C \in \mathcal{C}_{\mathcal{A}}$, we have $p_{C}^{a}(y) \neq 0, y \in Y$. We obtain (23).

Thus for a double stochastic matrix $\mathbf{P}^{b / a}$ we can choose

$$
\theta_{C}\left(b_{2}\right)=\pi-\theta_{C}\left(b_{1}\right)
$$

Proposition 1. Let the conditions of Lemma 2 hold true. Then the condition (22) holds true for any Kolmogorov model iff $\epsilon_{1}=-\epsilon_{2}$

Proof. By (24) we obtain:

$$
\Delta(C)=\left(\epsilon_{1}+\epsilon_{2}\right) \theta_{C}\left(b_{1}\right)-\epsilon_{2} \pi
$$

Let us denote the unit sphere in the Hilbert space $E=\Phi(X, \mathbf{C})$ by the symbol $S$. The map $J^{b / a}: \mathcal{C} \rightarrow S$ need not be a surjection (injection), see examples in section 6 . In general the set of pure states corresponding to a Kolmogorovian model

$$
S_{\mathcal{C}} \equiv S_{\mathcal{C}}^{b / a}=J^{b / a}(\mathcal{C})
$$

is just a proper subset of the sphere $S$. The structure of the set of pure states $S_{\mathcal{C}}$ is determined by the Kolmogorov model.

We remark that for a double stochastic matrix $\mathbf{P}^{b / a}$ (and $\epsilon_{1}=-\epsilon_{2}$ ) the condition (22) does not depend on the set $\mathcal{C}$ (i.e., a Kolmogorov model). Here always $\Delta=\pi$. We also remark that, in fact, only double stochastic matrices 
$\mathbf{P}^{b / a}$ has such a property. By using calculations which have been done in the proof of Lemma 1 we obtain the following more general result.

Lemma 2a. Let $a$ and $b$ be incompatible random variables. Then for any context $C \in \mathcal{C}$ the following equality holds true:

$$
\cos \theta_{C}\left(b_{1}\right)=-k \cos \theta_{C}\left(b_{2}\right)
$$

where

$$
k \equiv k^{b / a}=\sqrt{\frac{p_{12} p_{22}}{p_{11} p_{21}}}
$$

Proposition 2. Let $k>0$ be a real number and let angles $\theta_{1}, \theta_{2} \in[0, \pi]$ be connected by (22). If for all $\theta_{2} \in[0, \pi]$

$$
\cos \theta_{1}=-k \cos \theta_{2}
$$

then $k=1$ and $\Delta=\pi$.

Proof. By (22) we have $\theta_{1}=\epsilon_{1} \Delta+\epsilon_{1} \epsilon_{2} \theta_{2}$. Thus $\cos \left(\epsilon_{1} \Delta+\epsilon_{1} \epsilon_{2} \theta_{2}\right)=$ $-k \cos \theta_{2}$ for all $\theta_{2} \in[0, \pi]$. So $\cos \left(\Delta+\epsilon_{2} \theta_{2}\right)=-k \cos \theta_{2}$. Let $\theta_{2}=\epsilon_{2}(-\Delta+$ $\pi / 2)$. So $\cos (-\Delta+\pi / 2)=0$. Thus $\Delta=0$ or $\Delta=\pi$. Let $\Delta=0$. Then $\cos \theta=-k \cos \theta$ for any $\theta \in[0, \pi]$. This contradicts to positivity of $k$. So $\Delta=\pi$ and $k=1$. To get both $\theta_{1}, \theta_{2} \in[0, \pi]$ we should choose $\epsilon_{1}=-\epsilon_{2}$.

We also remark that $k^{b / a}=1$ iff $\mathbf{P}^{b / a}$ is double stochastic.

3.5. Extension of the Hilbert space representation map. The sets $A_{i}$ are not contexts with respect to $\mathcal{A}$, since $\mathbf{P}\left(A_{1} A_{2}\right)=0$. Thus $J^{b / a}\left(A_{i}\right)$ cannot be defined by (12). It is natural to extend the map $J^{b / a}$ to sets $A_{i}$ by setting

$$
J^{b / a}\left(A_{i}\right)=e_{i}^{a}, i=1,2 .
$$

We set

$$
\overline{\mathcal{C}}=\mathcal{C} \cup \mathcal{A}
$$

Thus we have constructed the Hilbert space representation:

$$
J^{b / a}: \overline{\mathcal{C}} \rightarrow S
$$

We set $S_{\overline{\mathcal{C}}}=J^{b / a} \overline{\mathcal{C}}$.

3.6. Nonsensitive contexts. Let $\delta\left(B_{i} / \mathcal{A}, C\right)=0, i=1,2$. So $\lambda\left(B_{i} / \mathcal{A}, C\right)=$ 0 and, hence, $\theta\left(B_{i} / \mathcal{A}, C\right)=\pi / 2$. Here $($ for $x \in X)$ :

$$
\varphi_{C}(x)=J^{b / a}(C)(x)=\sqrt{p_{C}^{a}\left(a_{1}\right) p\left(x / a_{1}\right)}+e^{i \epsilon(x) \frac{\pi}{2}} \sqrt{p_{C}^{a}\left(a_{2}\right) p\left(x / a_{2}\right)}
$$


Thus

$$
\varphi_{C}(x)=\sqrt{p_{C}^{a}\left(a_{1}\right) p\left(x / a_{1}\right)}+\epsilon(x) i \sqrt{p_{C}^{a}\left(a_{2}\right) p\left(x / a_{2}\right)}
$$

We set

$$
\mathcal{C}_{0}=\left\{C \in \mathcal{C}: \delta\left(B_{j} / \mathcal{A}, C\right)=0\right\}
$$

Contexts $C \in \mathcal{C}_{0}$ are said to be $b / a$-nonsensitive contexts. These are complexes of physical (or, e.g., social) conditions $C$ such that a measurement of $a$ under $C$ does not disturb the probability distribution of $b$. We remark that $\Omega$ always belong to $\mathcal{C}_{0}$. However, in general $\mathcal{C}_{0} \neq\{\Omega\}$, see section 6 .

3.7. Non injectivity of the Hilbert space representation map. Let $C_{1}, C_{2} \in \mathcal{C}$ be contexts such that probability distributions of random variables $a$ and $b$ under $C_{1}$ and $C_{2}$, respectively, coincide:

$$
p_{C_{1}}^{a}(y)=p_{C_{2}}^{a}(y), y \in Y, \quad p_{C_{1}}^{b}(x)=p_{C_{2}}^{b}(x), x \in X .
$$

In such a case $\delta\left(b=x / \mathcal{A}, C_{1}\right)=\delta\left(b=x / \mathcal{A}, C_{2}\right)$. Thus corresponding phases also coincide: $\theta\left(b=x / \mathcal{A}, C_{1}\right)=\theta\left(b=x / \mathcal{A}, C_{2}\right)$. Hence $\phi_{C_{1}}(x)=\phi_{C_{2}}(x), x \in$ $X$, and $J^{b / a}\left(C_{1}\right)=J^{b / a}\left(C_{2}\right)$, see section 6 for examples.

3.8. Nonquantum Hilbert space representations of Kolmogorovian models. Of course, for arbitrary random variables $a$ and $b$ the matrix $\mathbf{P}^{b / a}$ need not be double stochastic. Thus a representation of probabilities by vectors in a single Hilbert space we can obtain for a very restricted class of random variables. In particular, such random variables are considered in quantum theory (in the formalism of Dirac-von Neumann). In general, for each random variable we should introduce its own scalar product and corresponding Hilbert space:

$$
\begin{aligned}
& H_{b}=\left(E,(\cdot, \cdot)_{b}\right), H_{a}=\left(E,(\cdot, \cdot)_{a}\right), \ldots, \text { where } \\
& \qquad(\varphi, \psi)_{b}=\sum_{j} v_{j}^{b} \bar{w}_{j}^{b} \text { for } \varphi=\sum_{j} v_{j}^{b} e_{j}^{b}, \psi=\sum_{j} w_{j} e_{j}^{b},
\end{aligned}
$$

and

$$
(\varphi, \psi)_{a}=\sum_{j} v_{j}^{a} \bar{w}_{j}^{a} \text { for } \varphi=\sum_{j} v_{j}^{a} e_{j}^{a}, \psi=\sum_{j} w_{j}^{a} e_{j}^{a} .
$$

The Hilbert spaces $H_{b}, H_{a}, \ldots$ give the $b$-representation, the $a$-representation, $\ldots$ Thus $p_{C}^{b}\left(b_{j}\right)=\left|\left(\varphi, e_{j}^{b}\right)_{b}\right|^{2}$ and $p_{C}^{a}\left(a_{j}\right)=\left|\left(\varphi, e_{j}^{a}\right)_{a}\right|^{2}$ and so on. In the $H_{a}$ we have:

$$
E(a / C)=\sum_{y \in Y} y p_{C}^{a}(y)=a_{1}\left|\left(\varphi_{C}, e_{1}^{a}\right)_{a}\right|^{2}+a_{2}\left|\left(\varphi_{C}, e_{2}^{a}\right)_{a}\right|^{2}=\left(\hat{a} \varphi_{C}, \varphi_{C}\right)_{a}
$$


where the operator $\hat{a}: E \rightarrow E$ is determined by its eigenvectors: $\hat{a} e_{j}^{a}=a_{j} e_{j}^{a}$.

Of course, the representation of random variables by linear operators is just a convenient mathematical tool to represent the average of a random variable by using only the Hilbert space structure. We recall that we started with purely "classical" Kolmogorovian random variables.

As in the conventional quantum formalism we can also consider the map

$$
\tilde{J}^{b / a}: \overline{\mathcal{C}} \rightarrow \tilde{\Phi}(X, \mathbf{C}) .
$$

Here $\tilde{\Phi}(X, \mathbf{C})$ is the space of equivalent classes of functions under the equivalence relation: $\varphi$ equivalent $\psi$ iff $\varphi=t \psi, t \in \mathbf{C},|t|=1$, and $\tilde{J}^{b / a}(C)=$ $t \phi_{C}, t \in \mathbf{C},|t|=1$, where $C \in \overline{\mathcal{C}}$.

Conclusion. In the contextual probabilistic approach we can construct a natural map from the set of contexts into the unit sphere of the complex Hilbert space. Such a map is determined by a pair $a, b$ of incompatible random variables. Unitarity of the matrix $V^{b / a}$ of transition from the basis $\left\{e_{i}^{a}\right\}$ to the basic $\left\{e_{i}^{b}\right\}$ (these basis correspond to random variables a and $b$, respectively) is equivalent to the possibility of using Born's rule both in the $a$ and $b$ representations. In general (i.e., for an arbitrary set of contexts) such a construction can be realized only for double stochastic matrix of transition probabilities.

Everywhere below we restrict our considerations to the case in which the matrix of transition probabilities $\mathbf{P}^{b / a}$ is double stochastic.

\section{Noncommutativity of operators represent- ing Kolmogorovian random variables}

We consider in this section the case of real valued random variables. Here spectra of random variables $b$ and $a$ are subsets of $\mathbf{R}$.

We set $q_{1}=\sqrt{p_{11}}=\sqrt{p_{22}}$ and $q_{2}=\sqrt{p_{12}}=\sqrt{p_{21}}$.

Thus the vectors of the $a$-basis, see (19), have the following form:

$$
e_{1}^{a}=\left(q_{1}, q_{2}\right), \quad e_{2}^{a}=\left(e^{i \epsilon_{1} \theta_{1}} q_{2}, e^{i \epsilon_{2} \theta_{2}} q_{1}\right) .
$$

Since $\theta_{1}+\theta_{2}=\pi$, we get $e_{2}^{a}=e^{i \epsilon_{2} \theta_{2}}\left(-q_{2}, q_{1}\right)$. The factor $e^{i \epsilon_{2} \theta_{2}}$ does not play any role in probabilistic considerations. Hence we can work in the new basis:

$$
e_{1}^{a}=\left(q_{1}, q_{2}\right), \quad e_{2}^{a}=\left(-q_{2}, q_{1}\right)
$$


We now find matrices of operators $\hat{a}$ and $\hat{b}$ in the $b$-representation. The latter one is diagonal. For $\hat{a}$ we have: $\hat{a}=V \operatorname{diag}\left(\mathrm{a}_{1}, \mathrm{a}_{2}\right) \mathrm{V}^{\star}$, where $v_{11}=$ $v_{22}=q_{1}, v_{21}=-v_{12}=q_{2}$. Thus

$$
a_{11}=a_{1} q_{1}^{2}+a_{2} q_{2}^{2}, a_{22}=a_{1} q_{2}^{2}+a_{2} q_{1}^{2}, a_{12}=a_{21}=\left(a_{1}-a_{2}\right) q_{1} q_{2} .
$$

Hence

$$
[\hat{b}, \hat{a}]=\hat{m}
$$

where $m_{11}=m_{22}=0$ and $m_{12}=-m_{21}=\left(a_{1}-a_{2}\right)\left(b_{2}-b_{1}\right) q_{1} q_{2}$. Since $a_{1} \neq a_{2}, b_{1} \neq b_{2}$ and $q_{j} \neq 0$, we have $\hat{m} \neq 0$.

\section{The role of simultaneous double stochastic- ity of $\mathbf{P}^{b / a}$ and $\mathbf{P}^{a / b}$}

Starting with the $b$-representation - complex amplitudes $\phi_{C}(x)$ defined on the spectrum (range of values) of a random variable $b$ - we constructed the $a$-representation. This construction is natural (i.e., reproduce Born's probability rule) only in the case in which $\mathbf{P}^{b / a}$ is double stochastic. We would like to have a symmetric model. So by starting with the $a$-representation complex amplitudes $\phi_{C}(y)$ defined on the spectrum (range of values) of a random variable $a$ - we would like to construct the natural $b$-representation. Thus both matrices of transition probabilities $\mathbf{P}^{b / a}$ and $\mathbf{P}^{a / b}$ should be double stochastic.

Theorem 2. Let the matrix $\mathbf{P}^{b / a}$ be double stochastic. The contexts $B_{1}, B_{2}$ belong to $\mathcal{C}$ iff the matrix $\mathbf{P}^{a / b}$ is double stochastic.

Proof. We have

$$
\lambda\left(B_{2} / \mathcal{A}, B_{1}\right)=-\frac{\mu_{1}^{2}+\mu_{2}^{2}}{2 \mu_{1} \mu_{2}},
$$

where $\mu_{j}=\sqrt{p_{B_{1}}^{a}\left(a_{j}\right) p\left(b_{2} / a_{j}\right)}$. So $\lambda\left(B_{2} / \mathcal{A}, B_{1}\right) \geq 1$ and we have the trigonometric behaviour only in the case $\mu_{1}=\mu_{2}$. Thus: $p_{B_{1}}^{a}\left(a_{1}\right) p\left(b_{2} / a_{1}\right)=p_{B_{1}}^{a}\left(a_{2}\right) p\left(b_{2} / a_{2}\right)$. In this case $\lambda\left(B_{2} / \mathcal{A}, B_{1}\right)=-1$, so $\theta\left(B_{2} / \mathcal{A}, B_{1}\right)=\pi$, and consequently $\theta\left(B_{1} / \mathcal{A}, B_{1}\right)=0$. We pay attention to the fact that $p_{B_{i}}^{a}\left(a_{j}\right)=p^{a / b}\left(a_{j} / b_{i}\right) \equiv$ $p\left(a_{j} / b_{i}\right)$. Thus we have:

$$
p\left(a_{1} / b_{1}\right) p\left(b_{2} / a_{1}\right)=p\left(a_{2} / b_{1}\right) p\left(b_{2} / a_{2}\right)
$$


In the same way by using conditioning with respect to $B_{2}$ we obtain:

$$
p\left(a_{1} / b_{2}\right) p\left(b_{1} / a_{1}\right)=p\left(a_{2} / b_{2}\right) p\left(b_{1} / a_{2}\right) .
$$

By using double stochasticity of $\mathbf{P}^{b / a}$ we can rewrite the last equality as

$$
p\left(a_{1} / b_{2}\right) p\left(b_{2} / a_{2}\right)=p\left(a_{2} / b_{2}\right) p\left(b_{2} / a_{1}\right) .
$$

Thus by (29) and (30) we have:

$$
\frac{p\left(a_{1} / b_{2}\right)}{p\left(a_{2} / b_{1}\right)}=\frac{p\left(a_{2} / b_{2}\right)}{p\left(a_{1} / b_{1}\right)} .
$$

Hence $p\left(a_{1} / b_{2}\right)=t p\left(a_{2} / b_{1}\right)$ and $p\left(a_{2} / b_{2}\right)=t p\left(a_{1} / b_{1}\right), t>0$. But $1=p\left(a_{1} / b_{2}\right)+$ $p\left(a_{2} / b_{2}\right)=t\left[p\left(a_{2} / b_{1}\right)+p\left(a_{1} / b_{1}\right)\right]=t$.

To finish the proof we need the following well known result:

Lemma 3. Both matrices of transition probabilities $\mathbf{P}^{b / a}$ and $\mathbf{P}^{a / b}$ are double stochastic iff the transition probabilities are symmetric, i.e.,

$$
p\left(b_{i} / a_{j}\right)=p\left(a_{j} / b_{i}\right), i, j=1,2 .
$$

This is equivalent that random variables $a$ and $b$ have the uniform probability distribution: $p^{a}\left(a_{i}\right)=p^{b}\left(b_{i}\right)=1 / 2, i=1,2$.

This Lemma has important physical consequences. A natural (Bornian) Hilbert space representation of contexts can be constructed only on the basis of a pair of (incompatible) uniformly distributed random variables.

Lemma 4. Let both matrices $\mathbf{P}^{b / a}$ and $\mathbf{P}^{a / b}$ be double stochastic. Then

$$
\lambda\left(B_{i} / \mathcal{A}, B_{i}\right)=1 \text {. }
$$

Proof. Here $\delta\left(B_{i} / \mathcal{A}, B_{i}\right)=1-p\left(b_{i} / a_{1}\right) p\left(a_{1} / b_{i}\right)-p\left(b_{i} / a_{2}\right) p\left(a_{2} / b_{i}\right)=1-$ $p\left(a_{1} / b_{i}\right)^{2}-p\left(a_{2} / b_{i}\right)^{2}=2 p\left(a_{1} / b_{i}\right) p\left(a_{2} / b_{i}\right)$. Thus $\lambda\left(B_{i} / \mathcal{A}, B_{i}\right)=1$.

By (32) we have

$$
\lambda\left(B_{i} / \mathcal{A}, B_{j}\right)=-1, i \neq j,
$$

Thus

$$
\theta\left(B_{i} / \mathcal{A}, B_{i}\right)=0 \text { and } \theta\left(B_{i} / \mathcal{A}, B_{j}\right)=\pi, i \neq j .
$$

Proposition 2. Let both matrices of transition probabilities $\mathbf{P}^{b / a}$ and $\mathbf{P}^{a / b}$ be double stochastic. Then

$$
J^{b / a}\left(B_{j}\right)(x)=\delta\left(b_{j}-x\right), x \in X, \text { and } \quad J^{a / b}\left(A_{j}\right)(y)=\delta\left(a_{j}-y\right), y \in Y .
$$


Proof. Because $\theta\left(B_{1} / \mathcal{A}, B_{1}\right)=0$ we have:

$$
\begin{aligned}
J^{b / a}\left(B_{1}\right)\left(b_{1}\right)= & \sqrt{p\left(a_{1} / b_{1}\right) p\left(b_{1} / a_{1}\right)}+e^{i 0} \sqrt{p\left(a_{2} / b_{1}\right) p\left(b_{1} / a_{2}\right)} \\
& =p\left(a_{1} / b_{1}\right)+p\left(a_{2} / b_{1}\right)=1 .
\end{aligned}
$$

Because $\theta\left(B_{2} / \mathcal{A}, B_{1}\right)=\pi$ we have

$$
\begin{gathered}
J^{b / a}\left(B_{1}\right)\left(b_{2}\right)=\sqrt{p\left(a_{1} / b_{1}\right) p\left(b_{2} / a_{1}\right)}+e^{i \pi} \sqrt{p\left(a_{2} / b_{1}\right) p\left(b_{2} / a_{2}\right)} \\
=\sqrt{p\left(a_{1} / b_{1}\right)}\left(\sqrt{p\left(b_{2} / a_{1}\right.}-\sqrt{p\left(a_{2} / b_{1}\right)}\right)=0 .
\end{gathered}
$$

Thus in this case:

$$
J^{b / a}\left(B_{i}\right)=e_{i}^{b}, i=1,2 .
$$

\section{Example of the Hilbert space representa- tion of the contextual Kolmogorovian model}

We consider an example of a Kolmogorov probability space and a pair of dichotomous random variables $a, b$ which are incompatible. In this example the set of contexts with nontrivial disturbance term $\delta, \delta \neq 0$, is nonempty, so $\mathcal{C}_{0} \neq \mathcal{C}$.

6.1. Kolmogorov probability space and incompatible random variables. We find the image $S_{\mathcal{C}}$ of the set of contexts $\mathcal{C}$ in the Hilbert sphere $S \subset E=\Phi(X, C)$. In this example $S_{\mathcal{C}}$ is a proper subset of the sphere $S$. The Hilbert space representation map $J^{b / a}$ is not injective. Random variables $a$ and $b$ are represented by symmetric operators in the Hilbert space $E$. They do not commute.

Let $\Omega=\left\{\omega_{1}, \omega_{2}, \omega_{3}, \omega_{4}\right\}$ and $\mathbf{P}\left(\omega_{j}\right)=p_{j}>0, \sum_{j=1}^{4} p_{j}=1$. Let

$$
\begin{aligned}
& A_{1}=\left\{\omega_{1}, \omega_{2}\right\}, A_{2}=\left\{\omega_{3}, \omega_{4}\right\} \\
& B_{1}=\left\{\omega_{1}, \omega_{4}\right\}, B_{2}=\left\{\omega_{2}, \omega_{3}\right\}
\end{aligned}
$$

Let $p_{1}=p_{3}=q<\frac{1}{2}$ and $p_{2}=p_{4}=(1-2 q) / 2$. We denote this Kolmogorov probability space by the symbol $\mathcal{K}(q)$.

Here $\mathbf{P}\left(A_{1}\right)=\mathbf{P}\left(A_{2}\right)=\mathbf{P}\left(B_{1}\right)=\mathbf{P}\left(B_{2}\right)=\frac{1}{2}$. So the random variables $a$ and $b$ are uniformly distributed. Thus both matrices of transition probabilities $\mathbf{P}^{b / a}$ and $\mathbf{P}^{a / b}$ are double stochastic. Here

$$
\mathbf{P}^{b / a}=\mathbf{P}^{a / b}=\left(\begin{array}{lr}
2 q & 1-2 q \\
1-2 q & 2 q
\end{array}\right)
$$


We have the symmetry condition $\mathbf{P}\left(B_{i} / A_{j}\right)=\mathbf{P}\left(A_{j} / B_{i}\right)$.

6.2. Hilbert space representation of contexts. We choose $\epsilon_{1}=-1$ and $\epsilon_{2}=+1$ to fix the map $J^{b / a}$. We start with two-points contexts.

(a) Let $C=C_{24}=\left\{\omega_{2}, \omega_{4}\right\}$. Here $\mathbf{P}(C)=1-2 q, \mathbf{P}\left(B_{j} / C\right)=\mathbf{P}\left(A_{j} / C\right)=$ $\frac{1}{2}$. Thus $\delta=0$. By using the representation (27), we obtain:

$$
\varphi_{C_{24}}(x)=\left\{\begin{array}{l}
\sqrt{q}-i \sqrt{\frac{1-2 q}{2}}, x=b_{1} \\
\sqrt{\frac{1-2 q}{2}}+i \sqrt{q}, x=b_{2}
\end{array}\right.
$$

(b). Let $C=C_{13}=\left\{\omega_{1}, \omega_{3}\right\}$. Here everything is as in (a). So we have $\varphi_{C_{13}}=\varphi_{C_{24}}$ Thus $J^{b / a}$ is not injective: $J^{b / a}\left(C_{24}\right)=J^{b / a}\left(C_{13}\right)$.

(c) Let $C=C_{14}=\left\{\omega_{1}, \omega_{4}\right\}=B_{1}$. By general theory we have $\varphi_{C_{14}}(x)=$ $\delta\left(b_{1}-x\right)=e_{1}^{b}$. In the same way: $\varphi_{C_{23}}=\delta\left(b_{2}-x\right)=e_{2}^{b}$.

To find the Hilbert space representation of sets $C=C_{12}=\left\{\omega_{1}, \omega_{2}\right\}=A_{1}$ and $C=C_{34}=\left\{\omega_{3}, \omega_{4}\right\}=A_{2}$ we have to construct the basis $\left\{e_{j}^{a}\right\}$. We can choose:

$$
e_{1}^{a}=\left(\begin{array}{c}
\sqrt{2 q} \\
\sqrt{1-2 q}
\end{array}\right) \quad e_{2}^{a}=\left(\begin{array}{c}
-\sqrt{1-2 q} \\
\sqrt{2 q}
\end{array}\right)
$$

(d) Let $C=C_{123}=\left\{\omega_{1}, \omega_{2}, \omega_{3}\right\}$. Here $\mathbf{P}(C)=(2 q+1) / 2, \mathbf{P}\left(A_{1} / C\right)=$ $\mathbf{P}\left(B_{2} / C\right)=1 /(2 q+1), \mathbf{P}\left(A_{2} / C\right)=\mathbf{P}\left(B_{1} / C\right)=2 q /(2 q+1)$. Thus $\delta\left(B_{1} / \mathcal{A}, C\right)=$ $\frac{2 q(2 q-1)}{2 q+1}$ and, hence, $\lambda\left(B_{1} / \mathcal{A}, C\right)=-\frac{\sqrt{1-2 q}}{2}$. This context is trigonometric, i.e., the measurement of the random variable $a$ under the complex of physical conditions $C$ induces nontrivial, but relatively small statistical disturbance of the "b-property"; so $C_{123} \in \mathcal{C}$. We remark that $\lambda\left(B_{2} / \mathcal{A}, C\right)=\frac{\sqrt{1-2 q}}{2}$ (since $\mathbf{P}^{b / a}$ is double stochastic). ${ }^{11}$ We have:

$$
\varphi_{C_{123}}(x)= \begin{cases}\sqrt{\frac{2 q}{2 q+1}}-e^{i \arccos \frac{\sqrt{1-2 q}}{2}} \sqrt{\frac{2 q(1-2 q)}{2 q+1}}, x=b_{1} \\ \sqrt{\frac{1-2 q}{2 q+1}}+e^{i \arccos \frac{\sqrt{1-2 q}}{2}} \frac{2 q}{\sqrt{2 q+1}}, \quad x=b_{2}\end{cases}
$$

Remark. In principle, we could choose, e.g.,

$$
e_{2}^{a}=\left(\begin{array}{c}
-e^{i \theta} \sqrt{1-2 q} \\
e^{i \theta} \sqrt{2 q}
\end{array}\right), \theta=\arccos \frac{\sqrt{1-2 q}}{2} .
$$

\footnotetext{
${ }^{11}$ We pay attention on the dependence of $\theta=\arccos \frac{\sqrt{1-2 q}}{2}$ on the parameter $q: \theta(q)$ increases from $\pi / 3$ to $\pi / 2$, when $q$ increases from 0 to $1 / 2$.
} 
Thus

$$
\varphi_{C_{123}}=\frac{1}{\sqrt{2 q+1}} e_{1}^{a}+e^{i \arccos \frac{\sqrt{1-2 q}}{2}} \sqrt{\frac{2 q}{2 q+1}} e_{2}^{a} .
$$

(e) Let $C=C_{124}=\left\{\omega_{1}, \omega_{2}, \omega_{4}\right\}$. Here $\mathbf{P}(C)=1-q, \mathbf{P}\left(A_{1} / C\right)=\mathbf{P}\left(B_{1} / C\right)=$ $1 / 2(1-q), \mathbf{P}\left(A_{2} / C\right)=\mathbf{P}\left(B_{2} / C\right)=(1-2 q) / 2(1-q)$. Thus $\delta\left(B_{1} / \mathcal{A}, C\right)=$ $q(1-2 q) /(1-q)$ and, hence, $\lambda\left(B_{1} / \mathcal{A}, C\right)=\sqrt{\frac{q}{2}}<1$, and the context $C_{124} \in \mathcal{C}$. Thus:

$$
\begin{aligned}
& \varphi_{C_{124}}(x)=\left\{\begin{array}{l}
\sqrt{\frac{q}{1-q}}+e^{-i \arccos \sqrt{\frac{q}{2}}} \frac{1-2 q}{\sqrt{2(-q)}}, \quad x=b_{1} \\
\sqrt{\frac{1-2 q}{2(1-q)}}-e^{-i \arccos \sqrt{\frac{q}{2}}} \sqrt{\frac{q(1-2 q)}{1-q}}, x=b_{2}
\end{array}\right. \\
& \varphi_{C_{124}}(x)=\frac{1}{\sqrt{2(1-q)}} e_{1}^{a}-e^{-i \arccos \sqrt{\frac{q}{2}}} \sqrt{\frac{1-2 q}{2(1-q)}} e_{2}^{a} .
\end{aligned}
$$

(f) Let $C=C_{234}=\left\{\omega_{2}, \omega_{3}, \omega_{4}\right\}$. Here $\mathbf{P}(C)=1-q, \mathbf{P}\left(A_{1} / C\right)=\mathbf{P}\left(B_{1} / C\right)=$ $(1-2 q) / 2(1-q), \mathbf{P}\left(A_{2} / C\right)=\mathbf{P}\left(B_{2} / C\right)=1 / 2(1-q)$. Thus $\delta\left(B_{1} / \mathcal{A}, C\right)=$ $q(2 q-1) /(1-q)$ and, hence, $\lambda\left(B_{1} / \mathcal{A}, C\right)=-\sqrt{\frac{q}{2}}, \lambda\left(B_{2} / \mathcal{A}, C\right)=\sqrt{\frac{q}{2}}$. Here:

$$
\begin{aligned}
& \varphi_{C_{234}}(x)=\left\{\begin{array}{l}
\sqrt{\frac{q(1-2 q)}{1-q}}-e^{i \arccos \sqrt{\frac{q}{2}}} \sqrt{\frac{1-2 q}{2(1-q)}}, \quad x=b_{1} \\
\frac{1-2 q}{\sqrt{2(1-q)}}+e^{i \arccos \sqrt{\frac{q}{2}}} \sqrt{\frac{q}{1-q}}, \quad x=b_{2}
\end{array}\right. \\
& \varphi_{C_{234}}(x)=\sqrt{\frac{1-2 q}{2(1-q)}} e_{1}^{a}+e^{i \arccos \sqrt{\frac{q}{2}}} \frac{1}{\sqrt{2(1-q)}} e_{2}^{a} .
\end{aligned}
$$

(g) Let $C=C_{134}=\left\{\omega_{1}, \omega_{3}, \omega_{4}\right\}$. Here $\mathbf{P}(C)=(2 q+1) / 2, \mathbf{P}\left(A_{1} / C\right)=$ $\mathbf{P}\left(B_{2} / C\right)=2 q /(2 q+1), \mathbf{P}\left(A_{2} / C\right)=\mathbf{P}\left(B_{1} / C\right)=1 /(2 q+1)$. Thus $\delta\left(B_{1} / \mathcal{A}, C\right)=$ $2 q(1-2 q) /(2 q+1)$ and, hence, $\lambda\left(B_{1} / \mathcal{A}, C\right)=\frac{\sqrt{1-2 q}}{2}$. Thus:

$$
\begin{aligned}
\varphi_{C_{134}}(x) & = \begin{cases}\frac{2 q}{\sqrt{2 q+1}}+e^{-i \arccos \frac{\sqrt{1-2 q}}{2}} \sqrt{\frac{1-2 q}{2 q+1}}, & x=b_{1} \\
\sqrt{\frac{2 q(1-2 q)}{2 q+1}}-e^{-i \arccos \frac{\sqrt{1-2 q}}{2}} \sqrt{\frac{2 q}{2 q+1}}, x=b_{2}\end{cases} \\
\varphi_{C_{134}} & =\sqrt{\frac{2 q}{2 q+1}} e_{1}^{a}-e^{-i \arccos \frac{\sqrt{1-q}}{2}} \frac{1}{\sqrt{2 q+1}} e_{2}^{a} .
\end{aligned}
$$

(h) Let $C=\Omega$. Here we know from the beginning that $\delta\left(B_{j} / \mathcal{A}, C\right)=0$. Here $\mathbf{P}\left(A_{i} / C\right)=\mathbf{P}\left(A_{i}\right)=1 / 2$ and $\mathbf{P}\left(B_{i} / C\right)=\mathbf{P}\left(B_{i}\right)=1 / 2$. Thus $J^{b / a}(\Omega)=$ $J^{b / a}\left(C_{24}\right)=J^{b / a}\left(C_{13}\right)=($ (33) $)$. 
In this example the set of nonsensitive contexts contains three contexts: $\mathcal{C}_{0}=\left\{\Omega, C_{24}, C_{13}\right\}$. We have

$$
S_{\overline{\mathcal{C}}}=\left\{\varphi_{\Omega}, \varphi_{C_{14}}=e_{1}^{b}, \varphi_{C_{23}}=e_{2}^{b}, \varphi_{C_{12}}=e_{1}^{a}, \varphi_{C_{23}}=e_{2}^{a}, \varphi_{C_{124}}, \varphi_{C_{234}}, \varphi_{C_{123}}, \varphi_{C_{134}}\right\}
$$

So the set of pure states $S_{\overline{\mathcal{C}}}$ is a finite, five-points, subset of the unit sphere in the two dimensional Hilbert space.

We remark that there is a parameter $q \in(0,1 / 2)$ determining a Kolmogorov probability model $\mathcal{K}(q)$. For each value of $q$ we have a finite set of pure states. However, a family $\mathcal{K}(q), q \in(0,1 / 2)$, of Kolmogorov probability spaces generates a "continuous" set $\cup_{q} S_{\overline{\mathcal{C}}}(q)$ of pure states.

\section{Contextual correspondence between Kol- mogorovian random variables and quantum observables}

We begin with the following standard definition:

Definition 3. For a self-adjoint operator $\hat{d}$ the quantum mean value in the state $\varphi$ is defined by

$$
\langle\hat{d}\rangle_{\varphi}=(\hat{d} \varphi, \varphi) .
$$

Theorem 3. For any map $f: \mathbf{R} \rightarrow \mathbf{R}$ we have:

$$
\langle f(\hat{a})\rangle_{\varphi_{C}}=E(f(a) / C), \quad\langle f(\hat{b})\rangle_{\varphi_{C}}=E(f(b) / C)
$$

for any context $C \in \overline{\mathcal{C}}$.

Proof. By using Borness of the $b$-representation we obtain:

$$
E\left(f(b / C)=\sum_{x \in X} f(x) p_{c}^{b}(x)=\sum_{x \in X} f(x)\left|\left(\varphi_{C}, e_{x}^{b}\right)\right|^{2}=\langle f(\hat{b})\rangle_{\varphi_{C}}\right.
$$

The same result we have for the $f(\hat{a})$ since (as $\mathbf{P}^{b / a}$ is double stochastic) we have Born's probability rule both for $b$ and $a$.

Theorem 4. Let $f, g: \mathbf{R} \rightarrow \mathbf{R}$ be two arbitrary functions. Then

$$
E(f(a)+g(b) / C)=\langle f(\hat{a})+g(\hat{b})\rangle_{\varphi_{C}}
$$

for any context $C \in \overline{\mathcal{C}}$. 
Proof. By using linearity of the Kolmogorov mathematical expectation, Theorem 3, and linearity of the Hilbert space scalar product we obtain:

$$
\begin{gathered}
E(f(a(\omega))+g(b(\omega)) / C)=E(f(a(\omega) / C)+E(g(b(\omega)) / C) \\
=\langle f(\hat{a})\rangle_{\varphi_{C}}+\langle g(\hat{b})\rangle_{\varphi_{C}}=\langle f(\hat{a})+g(\hat{b})\rangle_{\varphi_{C}}
\end{gathered}
$$

Denote the linear space of all random variables of the form $d(\omega)=$ $f(a(\omega))+g(b(\omega))$ by the symbol $\mathcal{O}_{+}(a, b)$ and the linear space of operators of the form $\hat{d}=f(\hat{a})+g(\hat{b})$ by $\mathcal{O}_{+}(\hat{a}, \hat{b})$.

Theorem 5. The map $T=T^{a / b}: \mathcal{O}_{+}(a, b) \rightarrow \mathcal{O}_{+}(\hat{a}, \hat{b}), d=f(a)+g(b) \rightarrow$ $\hat{d}=f(\hat{a})+g(\hat{b})$, preserves the conditional expectation:

$$
\langle T(d)\rangle_{\varphi_{C}}=(T(d) J(C), J(C))=E(d / C) .
$$

The transformation $T$ preserves the conditional expectation for random variables $d \in \mathcal{O}_{+}(a, b)$. But in general we cannot expect anything more, since in general $T$ does not preserve probability distributions. The important problem is to extend the map $T$ for a larger class (linear space?) of Kolmogorovian random variables with preserving (34). It is natural to define (as we always do in the conventional quantum formalism):

$$
T(f)(\hat{a}, \hat{b})=f(\hat{a}, \hat{b})
$$

where $f(\hat{a}, \hat{b})$ is the pseudo differential operator with the Weyl symbol $f(a, b)$. We shall see that already for $f(a, b)=a b$ (so $f(\hat{a}, \hat{b})=(\hat{a} \hat{b}+\hat{b} \hat{a}) / 2)$ the equality (34) is violated.

We can consider the $b$ and the $a$ as discrete analogues of the position and momentum observables. The operators $\hat{b}$ and $\hat{a}$ give the Hilbert space (quantum) representation of these observables.

We also introduce an analogue of the energy observable:

$$
\mathcal{H}(\omega)=\frac{h}{2}\left[a^{2}(\omega)+V(b(\omega))\right]
$$

where $h>0$ is a constant and $V: \mathbf{R} \rightarrow \mathbf{R}$ is a map. The Hilbert space representation of this observable is given by the operator of energy (Hamiltonian)

$$
\hat{\mathcal{H}}=\frac{h}{2}\left(\hat{a}^{2}+V(\hat{b})\right)
$$


By Theorem 5 for contexts $C \in \overline{\mathcal{C}}$ the averages of the observables $\mathcal{H}(\omega)$ (Kolmogorovian) and $\hat{\mathcal{H}}$ (quantum) coincide:

$$
E(\mathcal{H}(\omega) / C)=\langle\mathcal{H}\rangle_{\varphi_{C}}
$$

However, as we shall see, probability distributions do not coincide:

Proposition 3. There exists context $C$ such that the probability distribution of the random variable $d(\omega)=a(\omega)+b(\omega)$ with respect to $C$ does not coincide with the probability distribution of the corresponding quantum observable $\hat{d}=\hat{a}+\hat{b}$ with respect to the state $\varphi_{C}$.

Proof. It suffices to present an example of such a context $C$. Take the context $C=C_{234}$ from section 6 . We consider the case: $a(\omega)= \pm \gamma, b(\omega)=$ $\pm \gamma, \gamma>0$; so $d(\omega)=-2 \gamma, 0,2 \gamma$. Corresponding Kolmogorovian probabilities can easily be found:

$$
p_{C}^{d}(-2 \gamma)=q /(1-q), \quad p_{C}^{d}(0)=(1-2 q) /(1-q), \quad p_{C}^{d}(2 \gamma)=0 .
$$

We now find the probability distribution of $\hat{d}$. To do this, we find eigenvalues and eigenvectors of the self-adjoint operator $\hat{d}$. We find the matrix of the operator $\hat{d}$ in the basis $\left\{e_{j}^{b}\right\}: d_{11}=-d_{22}=4 q \gamma$ and $d_{12}=d_{21}=2 \gamma \sqrt{2 q(1-2 q)}$. We have $k_{1,2}= \pm 2 \sqrt{2 q} \gamma$. Of course, the range of values of the quantum observable $\hat{d}$ differs from the range of values of the random variable $d$. However, this difference of ranges of values is not so large problem in this case. The random variable $d$ takes only two values, $-2 \gamma, 0$ with the probability one. Moreover, we can represent values of the quantum observable $\hat{d}$ as just an affine transform of values of the random variable $d$ :

$$
d_{\text {quantum }}=2 \sqrt{2 q} d-\gamma .
$$

In principle we can interpret such a transformation as representing some special measurement procedure. Thus in this example the problem with spectrum is not crucial. The crucial problem is that $d$ and $\hat{d}$ have different probability distributions.

Corresponding eigenvectors are

$$
\begin{aligned}
& e_{1}^{d}=\frac{1}{\sqrt{2(1-\sqrt{2 q})}}(-\sqrt{1-2 q}, \sqrt{2 q}-1) \\
& e_{2}^{d}=\frac{1}{\sqrt{2(1+\sqrt{2 q})}}(-\sqrt{1-2 q}, \sqrt{2 q}+1)
\end{aligned}
$$


Finally, we find (by using the expression for $\varphi_{C_{234}}$ which was found in section 6):

$$
\begin{aligned}
& p_{c}^{\hat{d}}\left(k_{1}\right)=\left|\left(\varphi_{C}, e_{1}^{d}\right)\right|^{2}=\frac{(1-\sqrt{2 q})(2+\sqrt{2 q})}{4(1-q)} \\
& p_{c}^{\hat{d}}\left(k_{2}\right)=\left|\left(\varphi_{C}, e_{2}^{d}\right)\right|^{2}=\frac{(1+\sqrt{2 q})(2-\sqrt{2 q})}{4(1-q)}
\end{aligned}
$$

Thus $d$ and $\hat{d}$ have essentially different probability distributions.

\section{Dispersion-free states}

1. Von Neumann. As originally stated by von Neumann, [5] the problem of hidden variables is to find whether dispersion free states exist in QM. He answered the question in the negative. The problem of the existence of dispersion free states as well as von Neumann's solution were the subject of great debates. We do not want to go into detail see, e.g., [8], [9]. In our contextual approach an analogue of this problem can be formulated as

\section{Do dispersion free contexts exist?}

The answer is the positive. In the example of section 6 . we can take any atom of the Kolmogorov probability space $\mathcal{K}_{q}$, e.g., $C=\left\{\omega_{1}\right\}$. Since, for any random variable $\xi$ on the Kolmogorov space $\mathcal{K}_{q}$, it has a constant value on such a $C$ the dispersion of $\xi$ under the context $C$ is equal to zero:

$$
D(\xi / C)=E\left[(\xi-E(\xi / C))^{2} / C\right]=0 .
$$

However, dispersion free contexts do not belong to the system $\overline{\mathcal{C}}$ of contexts which can be mapped by $J^{a / b}$ into the Hilbert space $H$. On the one hand, our contextual approach gives the possibility to have the realist viewpoint to QM. On the other hand, it does not contradict to the von Neumann as well as other "no-go" theorems. The mathematical representation of contexts (complexes of physical conditions) given by the quantum formalism it too rough to represent dispersion free contexts.

2. Kochen and Specker. In the model of Kochen and Specker on the set $\mathcal{L}_{s}$ of self-adjoint operators there was considered only the structure of a partial algebra:

Products and sums are defined only for pairs of commutative operators.

A necessary condition for the existence of a hidden variable interpretation is then the existence of an embedding of the partial algebra $\mathcal{L}_{s}$ into 
a commutative algebra. Kochen and Specker proved that such an embedding is impossible for Hilbert spaces of dimension $\geq 3$. Despite the fact that we restricted our contextual considerations to representations of Kolmogorovian spaces based on pairs of (incompatible) dichotomous variables, we can compare our approach with Kochen-Specker approach. In our contextual approach the commutative algebra should be chosen as the algebra $R V(\Omega, \mathcal{F}, \mathbf{P})$ of random variables. But the whole formulation of the problem of embedding of $\mathcal{L}_{s}$ into $R V(\Omega, \mathcal{F}, \mathbf{P})$ is meaningless in our framework. The use of only the structure of prealgebra does not change anything. The operator $\hat{d}=\hat{a}+\hat{b} \in \mathcal{L}_{s}$ and it has the natural preimage $d=a+b \in R V(\Omega, \mathcal{F}, \mathbf{P})$. The problem is that $\hat{d}$ and $d$ can have different probability distributions.

Thus from our point of view the dimension of a Hilbert space is not important. Even, as we have seen, in the two-dimensional case only a very restricted class of variables $d \in R V(\Omega, \mathcal{F}, \mathbf{P})$ can be mapped into $\mathcal{L}_{s}$ with preserving of probability distributions.

\section{Classical and quantum spaces as rough im- ages of fundamental prespace}

Our contextual probabilistic model induces the following picture of physical reality.

1. Prespace and classical space. There exists a prespace $\Omega$ which points corresponds to primary (irreducible) states of physical systems, prestates or fundamental physical parameters. Functions $d: \Omega \rightarrow \mathbf{R}^{m}$ are said to be preobservables. The set of all preobservables is denoted by the symbol $\mathcal{O}_{p} \equiv \mathcal{O}_{p}(\Omega)$. We are not able (at least at the moment) to measure an arbitrary preobservable $d \in \mathcal{O}_{p}$.

Nevertheless, some preobservables can be measured. Suppose that there exists a preobservable $b$ such that all measurements can be reduced to some measurements of $b$, cf. L. De Broglie [10] on the possibility to reduce any measurement to a position measurement. Let $X \subset \mathbf{R}^{m}$ be the range of values ${ }^{12}$ of $b$. The $X$ is said to be a classical space ${ }^{13}$. Set $B_{x}=\{\omega \in \Omega: b(\omega)=$ $x\}=b^{-1}(x), x \in X$.

\footnotetext{
${ }^{12}$ See section 8.3 on some motivations to consider $X$ as a subset of $\mathbf{R}^{m}$.

${ }^{13}$ Of course, in such a model the classical space $X$ depends on the preobservable $X \equiv$ $X(b)$. Thus $X$ is the $b$-image of the prespace $\Omega$.
} 
In principle a set $B_{x}$ could contain millions of points. Dynamics in $X$ is classical dynamics. In our model, classical dynamics is a rough image of dynamics in the prespace $\Omega^{14}$.

2. Classical phase space. Let $a$ be a preobservable which is incompatible with our fundamental preobservable $b$ (space observable). We denote by $Y \subset \mathbf{R}^{m}$ the range of values of the $a$. The $Y$ is said to be conjugate space to the classical space $X$. We call the $b$ position and the a momentum. We set $A_{y}=\{\omega \in \Omega: a(\omega)=y\}=a^{-1}(y), y \in Y$.

Since $A_{y}$ is not a subset of $B_{x}$ for any $x \in X$, the point $y$ cannot be used to get finer description of any point $x \in X$. Thus by using values of the momentum we cannot obtain a finer space structure. The variables $b$ and $a$ are really incompatible. By fixing the value of, e.g., $a=y_{0}$ we cannot fix the value of $b=x_{0}$. It is important for future considerations to notice that sets $A_{y} B_{x}$ are not contexts (in the contextual(b,a)-picture). In general

$$
A_{y} B_{x} \notin \overline{\mathcal{C}}
$$

Remark. (Nonlocal dependence of incompatible variables at the prespace level). Since, for a fixed $y_{0} \in Y$, we have $A_{y_{0}} \cap B_{x} \neq \emptyset$ for any $x \in X$, a value $y_{0}$ of the momentum can be determined only by all values $x \in X$ of the position. Thus on the level of the prespace incompatible variables are nonlocally dependent. However, this prespace nonlocality could not be found in classical mechanics, since in the latter the finer prespace structure is destroyed by the rough $(x, y)$ encoding.

The space $\Pi=X \times Y \subset \mathbf{R}^{2 m}$ is a classical phase space. Dynamics in the phase space gives a rough image in the terms of the two incompatible variables of dynamics in the prespace. The phase space $\Pi$ is a classical contextual $(b, a)$-picture of the prespace $\Omega$. This picture is richer than the pure $b$-space picture. The $\Pi$ contains images of the two families of contexts $\mathcal{A}=\left\{A_{y}\right\}$ and $\mathcal{B}=\left\{B_{x}\right\}$.

3. On homogeneous structure of the classical space. In our probabilistic investigations we have seen that the most natural choice of incompatible variables corresponds to random variables $a(\omega)$ and $b(\omega)$ which are uniformly distributed. On the other hand, the creation of a uniform partition of the prespace $\Omega$ is the most natural way to create a rough image $X$ of the prespace - a classical space.

\footnotetext{
${ }^{14}$ Consider in the example of section 6 the trajectory $\omega_{1} \rightarrow \omega_{2} \rightarrow \omega_{3} \rightarrow \omega_{4} \rightarrow \omega_{1}$ in the $\Omega$. In the classical space $X$ this trajectory is represented by $b_{1} \rightarrow b_{1} \rightarrow b_{2} \rightarrow b_{2} \rightarrow b_{1}$.
} 
If a group of cognitive systems have used such a uniform partition of the prespace then the corresponding classical space should be homogeneous. This is a reason to assume (as we have done) that the classical space $X \subset \mathbf{R}^{m}$. But, of course, we could not deduce the real number structure of the classical space only on the basis of the fact that fundamental variables should be uniformly distributed.

4. Classical statistical mechanics. As the next step we can consider statistical mechanics on the classical space $X$. In such a statistical theory from the very beginning we lost the finer statistical structure of the model based on probability distributions on the prespace. Functions $u: \Pi \rightarrow \mathbf{R}^{q}$ are called classical observables. The set of classical observables is denoted by the symbol $\mathcal{O}_{c}(\Pi)$. We shall also use symbols $\mathcal{O}_{c}(X)$ and $\mathcal{O}_{c}(Y)$ to denote spaces of classical observables depending only on the $b$-position and the $a$ momentum, respectively.

5. Quantum mechanics and the Hilbert space representation of prespace contexts. Neither classical nor quantum mechanics can describe the individual dynamics in the prespace. Of course, such a viewpoint to quantum mechanics contradicts to the so called orthodox Copenhagen interpretation by which the wave function describes an individual quantum system. It seems that our contextual approach to quantum theory is closer to the so called statistical (or ensemble) interpretation of quantum mechanics. By the latter a wave function describes not an individual quantum system but statistical properties of an ensemble of quantum systems, see, e.g., [7].

By our contextual interpretation the wave function has a realist prespace interpretation. A complex amplitude is nothing than an image (induced by the contextual formula of total probability) of a set of fundamental parameters - context. Thus the Hilbert state space $H$ is not less real than the classical real space $\mathbf{R}^{3}$.

Observables which probability distributions can be found by using the representation by self-adjoint operators in the Hilbert space are called quantum observables. The set of quantum observables is denoted by the symbol $\mathcal{O}_{q}(H)$. Neither classical statistical nor quantum mechanics can provide knowledge about the probability distribution of an arbitrary preobservable. Nevertheless, the quantum theory gives some information about some preobservables, namely fundamental preobservable $b$ and $a$ and pre-observables $d$ belonging to the class $\mathcal{O}_{+}(a, b)$. Another way to look to the same problem is to say that the quantum theory (with our contextual probabilistic interpretation) gives the possibility to represent some prespace structures, namely 
some contexts $C \in \mathcal{C}$ by vectors of a Hilbert state space.

Neither classical nor quantum mechanics are fundamental theories. They could not give information about the point wise structure of the prespace $\Omega$. But the quantum formalism represents some complexes of physical conditions - domains in the prespace - which are not represented in the classical space or phase space. Of course, the quantum formalism also represents classical position states $x \in X$ by wave functions $\varphi_{B_{x}}$ (Hilbert states $e_{x}^{b}$ ). Classical states $x \in X$ are images of prespace contexts $B_{x}$. But the quantum formalism represents also some sets $C \subset \Omega$ which have no classical images (namely, images in $X$ or $\Pi$ ).

Example. In the example of section 6 we take the set $C=C_{123}=$ $\left\{\omega_{1}, \omega_{2}, \omega_{3}\right\}$. Neither $C \subset B_{1}$ nor $C \subset B_{2}$. This prespace domain $C$ can be described neither by the position $x=b_{1}$ nor $x=b_{2}$. The quantum state $\varphi_{C} \in S \subset H$ representing this domain of the prespace describes the superposition of the two classical states $x=b_{1}$ and $x=b_{2}$. Hence a physical system prepared under the complex physical conditions $C=C_{123}$ is (from the classical viewpoint) in the superposition of two different positions.

6. Heisenberg uncertainty principle. We now take the context $C=$ $A_{y}$ for some $y \in Y$. Here the momentum $a$ has the definite value. But $A_{y} \cap B_{x} \neq \emptyset$ for any $x \in X$. Hence the state $\varphi_{C}=e_{y}^{a} \in \mathcal{S} \subset H$ also corresponds to the superposition of two positions $x=b_{1}$ and $x=b_{2}$. This is nothing else than (the discrete analogue) the Heisenberg uncertainty principle. In the same way in any state with the definite position, $\varphi_{C}=$ $e_{x}^{b}, x \in X$, the momentum can not have the definite value.

Thus the Hilbert sphere $S$ contains images of the classical spaces $X$ and $Y$ (but not the phase space $\Pi$, see further considerations), $X \subset S$ and $Y \subset S$. But the Hilbert space contains also images of nonclassical domains $C \in \overline{\mathcal{C}}$. We remark that (depending on the model) only a part of the Hilbert sphere corresponds to some domains of the prespace. All other quantum states, $\varphi \notin S_{\overline{\mathcal{C}}}$, are just ideal mathematical objects which do no correspond to any context in the prespace.

As was already remarked, the phase space $\Pi$ is not imbedded into the Hilbert sphere $S$, since contexts $C_{x y}=B_{x} A_{y}$ corresponding to points of the $\Pi$ do not belong to the system $\overline{\mathcal{C}}$ which is mapped into $S$.

7. Preobservables and quantum observables. For what class of preobservables can we find probability distributions with respect to contexts $C \in \overline{\mathcal{C}}$ by using the quantum formalism? As we have seen, we are not able to find the probability distribution for an arbitrary $d \in \mathcal{O}_{p}(\Omega)$. In general the 
operators $\hat{d}=d(\hat{a}, \hat{b})$ corresponding to functions $d(x, y)$ (e.g., $d(x, y)=x y$ or $d(x, y)=x+y)$ are not directly related to prequantum observables $d(\omega)=$ $d(b(\omega), a(\omega))$.

Only quantum observables $\hat{d}=f(\hat{b})$ and $\hat{d}=g(\hat{a})$ have the same probability distributions as the corresponding preobservables $d(\omega)=f(b(\omega))$ and $d(\omega)=g(a(\omega))$. By Theorem? the average is preserved by the canonical map $T^{b / a}: \mathcal{O}_{+}(a, b) \rightarrow \mathcal{O}_{+}(\hat{a}, \hat{b})$.

However, even such quantum observables give just a rough image of corresponding preobservables. By using quantum probabilistic formalism we can find probability distributions only for quantum states $\varphi_{C} \in S_{\overline{\mathcal{C}}} \subset H$. Those quantum states represent only some special contexts. Hence by using the quantum formalism we could not find the probability distribution of a preobservable $a(\omega)$ or $b(\omega)$ for an arbitrary context represented by a domain in the prespace $\Omega$. Neither we can reconstruct maps $a(\omega)$ and $b(\omega)$. Thus the quantum theory is not a fundamental theory. It does not provide the complete (even statistical) description of the prespace reality. However, some statistical information about the prespace structure can be obtained by using the quantum probabilistic formalism.

8. On the mystery of operator quantization. The origin of the operator quantization was always mysterious for me. Why the correspondence between functions and functions of operators (of the position and the momentum) provides the correct statistical description of quantum measurements? Our contextual model tells that the only reason is the coincidence of quantum averages with 'real' prespace (contextual) averages for some preobservables (in particular, of the form $f(b)+g(a)$ ).

Theorem 4 is only a sufficient condition for the coincidence of averages. But even such a result gives the possibility to connect the quantun Hamiltonian

$$
\hat{\mathcal{H}}=\frac{\hat{a}^{2}}{2}+V(\hat{b})
$$

with the realist preobservable $\mathcal{H}(a(\omega), b(\omega))=\frac{a(\omega)^{2}}{2}+V(b(\omega))$. Quantum averages of energy expressed by the Hilbert space averages of the Hamiltonian $\hat{\mathcal{H}}$ coincides with averages of the realist energy preobservable $\mathcal{H}(\omega)$. However, for some contexts $C$ quantum energy observable $\hat{\mathcal{H}}$ and energy preobservable $\mathcal{H}$ have different probability distributions, see Proposition 3.

The classical space is a contextual image of the fundamental prespace $\Omega$. This is a very poor image since only a few special contexts namely space- 
contexts have images in the classical space $\mathbf{R}^{3}$. In principle, there might be created various classical spaces (corresponding to various fundamental variables) on the basis of the prespace $\Omega$. Human beings have been creating their own (very special) classical space. Since light rays play the fundamental role in the creating of our classical space it can be called electromagnetic classical space. So the electromagnetic classical space is created on the basis on electromagnetic reduction of information. In principle there can exist systems which are able to perform some other reductions of information, e.g., gravitation reduction. They would create a gravitational classical space.

\section{Appendix on incompatible random vari- ables}

Proposition 4. Let $\left\{A_{j}\right\}$ and $\left\{B_{k}\right\}$ be two families of subsets of some set $\Omega$ and $\Omega=\cup_{j} A_{j}=\cup_{k} B_{k}$ and let

$$
A_{j} B_{k} \neq \emptyset
$$

for any pair $(j, k)$. Then

$$
\text { Neither } A_{j} \subset B_{k} \text { nor } B_{k} \subset A_{j}
$$

for any pair $(j, k)$. If $n=2$ then conditions (35) and (36) are equivalent.

Proof. Let (35) hold true. Suppose that there exists $(j, k)$ such that $A_{j} \subset B_{k}$. Thus we should have $A_{j} B_{i}=\emptyset$ for any $i \neq k$. Let (36) hold true and let $n=2: \mathcal{A}=\left\{A_{1}, A_{2}=\Omega \backslash A_{1}\right\}$ and $\mathcal{B}=\left\{B_{1}, B_{2}=\Omega \backslash B_{1}\right\}$. Suppose that, e.g., $A_{1} B_{1}=\emptyset$. Then we should have $A_{1} \subset B_{2}$.

If $n \neq 3$ then in general the condition (36) does not imply the condition (35). We can consider the following example. Let $\Omega=\left\{\omega_{1}, \ldots, \omega_{7}\right\}$ and let $A_{1}=\left\{\omega_{1}, \omega_{2} \omega_{3}\right\}, A_{2}=\left\{\omega_{4}, \omega_{5}\right\}, A_{3}=\left\{\omega_{6}, \omega_{7}\right\}$ and $B_{1}=\left\{\omega_{1}, \omega_{4}\right\}, B_{2}=$ $\left\{\omega_{2}, \omega_{5}, \omega_{6}\right\}, B_{3}=\left\{\omega_{3}, \omega_{7}\right\}$. Here (36) holds true but $A_{2} B_{3} \neq \emptyset$.

Finally, we remark that we have investigated only the case of dichotomous random variables. The general case is essentially more complicated from the mathematical viewpoint. In particular, not every double stochastic matrix can be represented as the square of a unitary matrix and so on... But I think that from the phemenological viewpoint the case of dichotomous observables is the most important, cf., e.g., Mackey [24] and the general quantum logic approach. 
I would like to thank L. Ballentine, S. Gudder, A. Holevo, P. Lahti, B Hiley, S. Goldstein, C. Fuchs, A. Peres, I. Volovich, R. Gill, J. Bub, T. Maudlin, H. Rauch, G. Emch, V. Belavkin, I. Helland for discussions on probabilistic foundations of quantum theory.

\section{References}

[1] A. Einstein, B. Podolsky, N. Rosen, Phys. Rev., 47, 777-780 (1935).

[2] J. S. Bell, Speakable and unspeakable in quantum mechanics. Cambridge Univ. Press (1987).

[3] A. Yu. Khrennikov, I.V. Volovich, Quantum Nonlocality, EPR Model, and Bell's Theorem, Proc. 3nd Sakharov Conf., Moscow, 2002, World Sci., 2, pp.269-276 (2003).

[4] J. Bell, Rev. Mod. Phys., 38, 447-452 (1966).

[5] J. von Neumann, Mathematical foundations of quantum mechanics (Princeton Univ. Press, Princeton, N.J., 1955).

[6] S. Kochen and E. Specker, J. Math. Mech., 17, 59-87 (1967).

[7] L. E. Ballentine, Rev. Mod. Phys., 42, 358-381 (1970).

[8] A. S. Wightman, Hilbert's sixth problem: mathematical treatment of the axioms of physics. Proc. Symposia in Pure Math., 28, 147-233 (1976).

[9] A. S. Holevo, Statistical structure of quantum theory. Springer, BerlinHeidelberg (2001).

[10] L. De Broglie, The current interpretation of wave mechanics, critical study. Elsevier Publ., Amsterdam-London-New York (1964).

[11] D. Bohm, Quantum theory, Prentice-Hall. Englewood Cliffs, NewJersey (1951).

[12] D. Bohm and B. Hiley, The undivided universe: an ontological interpretation of quantum mechanics. Routledge and Kegan Paul, London (1993).

[13] E. Nelson, Quantum fluctuation, Princeton Univ. Press, Princeton (1985).

[14] A. Yu. Khrennikov, Linear representations of probabilistic transformations induced by context transitions. J. Phys.A: Math. Gen., 34, 9965-9981 (2001). quant-ph/0105059

[15] A . Yu. Khrennikov, Quantum statistics via perturbation effects of preparation procedures. Il Nuovo Cimento, B 117, N. 3, 267-281 (2002).

A. Yu Khrennikov, Contextual viewpoint to quantum statistics, hep-th/0112076

[16] A. Yu. Khrennikov, Ensemble fluctuations and the origin of quantum probabilistic rule. J. Math. Phys., 43, N. 2, 789-802 (2002).

[17] A. Yu. Khrennikov, Unification of classical and quantum probabilistic formalisms. quant-ph/0302194. 
[18] D. Hilbert, J. von Neumann, L. Nordheim, Math. Ann., 98, 1-30 (1927).

[19] A. Lande, Foundations of quantum theory. Yale Univ. Press (1955).

A. Lande, New foundations of quantum mechanics. Cambridge Univ. Press, Cambridge.

[20] S. P. Gudder, Special methods for a generalized probability theory. Trans. AMS, 119, 428-442 (1965).

S. P. Gudder, Axiomatic quantum mechanics and generalized probability theory. Academic Press, New York (1970).

S. P. Gudder, An approach to quantum probability. Proc. Conf. Foundations of Probability and Physics, ed. A. Khrennikov. Quantum Prob. White Noise Anal., 13, 147-160, WSP, Singapore (2001).

[21] L. Accardi, The probabilistic roots of the quantum mechanical paradoxes. The wave-particle dualism. A tribute to Louis de Broglie on his 90th Birthday, ed. S. Diner, D. Fargue, G. Lochak and F. Selleri (D. Reidel Publ. Company, Dordrecht, 297-330, 1984);

L. Accardi, Urne e Camaleoni: Dialogo sulla realta, le leggi del caso e la teoria quantistica. Il Saggiatore, Rome (1997).

[22] L. E. Ballentine, Quantum mechanics (Englewood Cliffs, New Jersey, 1989).

[23] L. E. Ballentine, Interpretations of probability and quantum theory. Proc. Conf. Foundations of Probability and Physics, ed. A. Khrennikov. Q. Prob. White Noise Anal., 13, 71-84, WSP, Singapore (2001)

[24] G. W. Mackey, Mathematical foundations of quantum mechanics. W. A. Benjamin INc, New York (1963).

[25] G. Ludwig, Foundations of quantum mechanics (Springer, Berlin, 1983).

[26] E. B. Davies, J. T. Lewis, Comm. Math. Phys., 17, 239-260 (1970).

[27] A. Yu. Khrennikov, Interpretations of probability (VSP Int. Publ., Utrecht, 1999).

[28] L. Hardy, Quantum theory from intuitively reasonable axioms. Proc. Conf. Quantum Theory: Reconsideration of Foundations, ed. A. Khrennikov. Ser. Math. Modelling, 2, 117-130, Växjö Univ. Press (2002).

[29] A. N. Kolmogoroff, Grundbegriffe der Wahrscheinlichkeitsrech Springer Verlag, Berlin (1933); reprinted: Foundations of the Probability Theory. Chelsea Publ. Comp., New York (1956);

[30] A. Yu. Khrennikov, On foundations of quantum theory. Proc. Int. 
Conf. Quantum Theory: Reconsideration of Foundations. Ser. Math. Modelling in Phys., Engin., and Cogn. Sc., 163-196, Växjö Univ. Press, 2002. 